\title{
GENERALISED REGULAR VARIATION OF ARBITRARY ORDER
}

\author{
EDWARD OMEY \\ Mathematics and Statistics, Hogeschool-Universiteit Brussel \\ Affiliated Researcher ETEW, Katholieke Universiteit Leuven \\ Stormstraat 2, B-1000 Brussels, Belgium \\ E-mail: Edward.Omey@hubrussel.be \\ JOHAN SEGERS \\ Institut de Statistique, Biostatistique et Sciences Actuarielles (ISBA) \\ Université Catholique de Louvain \\ Voie du Roman Pays 20, B-1348 Louvain-la-Neuve, Belgium \\ E-mail: Johan.Segers@uclouvain.be
}

\begin{abstract}
Let $f$ be a measurable, real function defined in a neighbourhood of infinity. The function $f$ is said to be of generalised regular variation if there exist functions $h \not \equiv 0$ and $g>0$ such that $f(x t)-f(t)=h(x) g(t)+o(g(t))$ as $t \rightarrow \infty$ for all $x \in(0, \infty)$. Zooming in on the remainder term $o(g(t))$ eventually leads to the relation $f(x t)-f(t)=h_{1}(x) g_{1}(t)+\cdots+$ $h_{n}(x) g_{n}(t)+o\left(g_{n}(t)\right)$, each $g_{i}$ being of smaller order than its predecessor $g_{i-1}$. The function $f$ is said to be generalised regularly varying of order $n$ with rate vector $\boldsymbol{g}=\left(g_{1}, \ldots, g_{n}\right)^{\prime}$. Under general assumptions, $\boldsymbol{g}$ itself must be regularly varying in the sense that $\boldsymbol{g}(x t)=x^{\boldsymbol{B}} \boldsymbol{g}(t)+o\left(g_{n}(t)\right)$ for some upper triangular matrix $\boldsymbol{B} \in \mathbb{R}^{n \times n}$, and the vector of limit functions $\boldsymbol{h}=\left(h_{1}, \ldots, h_{n}\right)$ is of the form $\boldsymbol{h}(x)=\boldsymbol{c} \int_{1}^{x} u^{B} u^{-1} \mathrm{~d} u$ for some row vector $\boldsymbol{c} \in \mathbb{R}^{1 \times n}$. The uniform convergence theorem continues to hold. Based on this, representations of $f$ and $\boldsymbol{g}$ can be derived in terms of simpler quantities. Moreover, the remainder terms in the asymptotic relations defining higherorder regular variation admit global, non-asymptotic upper bounds.
\end{abstract}

1. Introduction. The aim of this paper is to provide an analysis of the asymptotic relation

$$
f(x t)=f(t)+\sum_{i=1}^{n} h_{i}(x) g_{i}(t)+o\left(\left|g_{n}(t)\right|\right), \quad x \in(0, \infty), t \rightarrow \infty .
$$

2010 Mathematics Subject Classification: Primary 26A12; Secondary 39B22, 60G70.

Key words and phrases: matrix exponential function, Potter bounds, rate vector, slow variation, uniform convergence theorem.

The paper is in final form and no version of it will be published elsewhere. 
Here $f$ and $g_{1}, \ldots, g_{n}$ are real-valued functions defined on a neighbourhood of infinity; the functions $g_{i}$ are eventually of constant sign and satisfy $g_{i}(t)=o\left(\left|g_{i-1}(t)\right|\right)$ as $t \rightarrow \infty$ for $i \in\{2, \ldots, n\}$; and $h_{1}, \ldots, h_{n}$ are real-valued functions defined on $(0, \infty)$. The functions $g_{1}, \ldots, g_{n}$ are called the rate functions and the functions $h_{1}, \ldots, h_{n}$ are called the limit functions.

The following observations are immediate. First, equation 1.1 subsumes the same equation with $n$ replaced by $k \in\{1, \ldots, n\}$ : the relation for $n$ can be seen as the one for $n-1$ plus an explicit remainder term. Second, the limit function $h_{1}$ is determined by $f$ and $g_{1}$, and the limit function $h_{i}(i \in\{2, \ldots, n\})$ is determined by $f$ and $g_{1}, \ldots, g_{i}$ and $h_{1}, \ldots, h_{i-1}$.

Equation (1.1) is closely related to a similar looking relation for the auxiliary functions $g_{i}$ : for each $i \in\{1, \ldots, n\}$,

$$
g_{i}(x t)=\sum_{j=i}^{n} A_{i j}(x) g_{j}(t)+o\left(\left|g_{n}(t)\right|\right), \quad x \in(0, \infty), t \rightarrow \infty .
$$

The functions $A_{i j}, 1 \leqslant i \leqslant j \leqslant n$, are real-valued functions on $(0, \infty)$. They are uniquely determined by the functions $g_{1}, \ldots, g_{n}$, and 1.2 implies the same equation for $n$ replaced by $k \in\{i, \ldots, n\}$.

Equations (1.1) and 1.2 fit into the theory of regular variation in the following way. Recall that a positive, measurable function $F$ defined in a neighbourhood of infinity is called regularly varying if for all $x \in(0, \infty)$ the limit $H(x)=\lim _{t \rightarrow \infty} F(x t) / F(t)$ exists in $(0, \infty)$ (Karamata, 1930, 1933). If $H \equiv 1$, then $F$ is called slowly varying. Setting $f=\log F$ and $h=\log H$ yields $h(t)=\lim _{t \rightarrow \infty}\{f(x t)-f(t)\}$, which is the case $n=1$ of (1.1) with $g_{1} \equiv 1$. If $F$ is measurable, then necessarily $H(x)=x^{c}$ for some $c \in \mathbb{R}$, and thus $h(x)=c \log (x)$. The case $n=1$ of (1.1) with general $g_{1}$ and $h(x)=c \int_{1}^{x} u^{b-1} \mathrm{~d} u$ was introduced in de Haan (1970) and is called extended or generalized regular variation, the symbol $\Pi$ being usually reserved for the class of functions $f$ for which $h(x)=c \log (x)$ with $c \neq 0$. When studying the rate of convergence for $\Pi$-varying functions, one naturally arrives at the case $n=2$ in 1.1), studied in Omey and Willekens (1988) and de Haan and Stadtmüller (1996). The next step, $n=3$, was the subject of Fraga Alves et al. (2006). The jump to arbitrary $n$ was made in Wang and Cheng (2005), where the focus is on the form of the limit functions $h_{i}$.

A minimal amount of regularity of the function $f$ needs to be presupposed for a rich enough theory to result. In this paper, we are content to assume $f$ that is Lebesgue measurable. As in Bingham et al. (1987, Chapter 1), an alternative would have been to start from the Baire property. According to Bingham and Ostaszewski (2009), truly minimal conditions can be formulated using infinite combinatorics.

Main references to the theory of regular variation, its extensions and its applications are the monographs by Seneta (1976), Bingham et al. (1987), and Geluk and de Haan (1987). In addition to the already cited papers, extensions of regular variation are studied in particular in de Haan (1974), Bingham and Goldie (1982a b), and Goldie and Smith (1987). Regular variation plays an important role in certain areas of probability theory, more precisely in the theory of domains of attraction of stable and max-stable 
distributions: see Feller (1971), Resnick (1987), and Meerschaert and Scheffler (2001). Higher-order extensions are relevant for instance to rates of convergence and Edgeworth expansions related to domains of attractions of max-stable distributions: see de Haan and Resnick (1996), Cheng and Jiang (2001), Wang and Cheng (2006), and Haeusler and Segers (2007).

1.1. Contributions. Our study is then centered around the following questions:

- If (1.1) and (1.2) are true for some $x \in(0, \infty)$, then when do they hold for all $x \in(0, \infty)$ ?

- What is the relation between (1.1) and (1.2)? When does (1.1) imply 1.2 ?

- What do the limit functions $h_{i}$ and $A_{i j}$ look like? How are they related?

- When are (1.1) and (1.2) (locally) uniform in $x$ ?

- Do the remainder terms in (1.1) and 1.2 admit global, non-asymptotic upper bounds?

- Can we represent $f$ in terms of $g_{1}, \ldots, g_{n}$ ? Can we retrieve $g_{1}, \ldots, g_{n}$ from $f$ ?

The key to the answers to these questions is a representation in terms of vectors and matrices. For instance, 1.2 can be written as

$$
\boldsymbol{g}(x t)=\boldsymbol{A}(x) \boldsymbol{g}(t)+o\left(\left|g_{n}(t)\right|\right), \quad x \in(0, \infty), t \rightarrow \infty ;
$$

here $\boldsymbol{g}=\left(g_{1}, \ldots, g_{n}\right)^{\prime}$ is a column-vector valued function, and $\boldsymbol{A}=\left(A_{i j}\right)_{i, j=1}^{n}$ is an upper-triangular matrix valued function. The function $\boldsymbol{A}$ necessarily satisfies the matrix version of the (multiplicative) Cauchy functional equation: $\boldsymbol{A}(x y)=\boldsymbol{A}(x) \boldsymbol{A}(y)$. Under measurability, this equation can be solved, yielding

$$
\boldsymbol{A}(x)=x^{B}, \quad x \in(0, \infty),
$$

for some upper triangular matrix $\boldsymbol{B} \in \mathbb{R}^{n \times n}$; here $x^{\boldsymbol{B}}=\exp \{(\log x) \boldsymbol{B}\}$, the exponential function of a matrix being defined by the usual series expansion. The rate vector $\boldsymbol{g}$ will be called regularly varying with index matrix $\boldsymbol{B}$; notation $\boldsymbol{g} \in R V_{\boldsymbol{B}}$. The row vector of limit functions $\boldsymbol{h}=\left(h_{1}, \ldots, h_{n}\right)$ in 1.1$)$ can then be written as

$$
\boldsymbol{h}(x)=\boldsymbol{c} \int_{1}^{x} u^{B} u^{-1} \mathrm{~d} u, \quad x \in(0, \infty),
$$

for some $\boldsymbol{c} \in \mathbb{R}^{1 \times n}$. We say that $f$ is generalized regularly varying with rate vector $\boldsymbol{g}$ and $\boldsymbol{g}$-index $\boldsymbol{c}$; notation $f \in G R V(\boldsymbol{g})$.

Conceptually, the $n$th order case is strongly similar to the first-order case provided we are ready to think of the index of regular variation as a matrix. The fact that this matrix is upper triangular comes from the assumption that $g_{i}(t)=o\left(\left|g_{i-1}(t)\right|\right)$ and gives rise to some convenient simplifications. The diagonal elements of $\boldsymbol{B}$ are at the same time the eigenvalues of $\boldsymbol{B}$ and the indices of regular variation of the functions $\left|g_{i}\right|$, and they determine the nature of 1.1 .

1.2. Outline. The structure of the body of our paper is as follows. In Section 2 we investigate some basic properties of the limit functions $A_{i j}$ and $h_{i}$ and of the sets of $x$-values for which the asymptotic relations 1.1 and 1.2 hold. We do not yet assume that $f$ or $\boldsymbol{g}$ are measurable. This assumption is added in Section 3, at which point we can 
define regularly varying rate vectors and generalized regular variation of arbitrary order. Two characterization theorems state the precise form of the limit functions $\boldsymbol{A}$ and $\boldsymbol{h}$ in terms of an index matrix $\boldsymbol{B}$ and an index vector $\boldsymbol{c}$.

The fundamental result in any branch of regular variation theory is the uniform convergence theorem. This is provided in Section 4 together with a first application, the representation of regularly varying rate vectors and of generalized regularly varying functions in terms of integrals of simpler quantities. In the Potter bounds of Section 5 , both the largest and the smallest eigenvalue of the index matrix $\boldsymbol{B}$ play a role.

Finally, two special cases are described in Section 6. First, if all eigenvalues (diagonal elements) of $\boldsymbol{B}$ are different from zero, then $f$ is essentially just a linear combination of the rate functions $g_{1}, \ldots, g_{n}$ (Section 6.1). Second, if all eigenvalues of $\boldsymbol{B}$ are distinct, then $f$ is essentially a linear combination of power functions, a logarithm, and the final rate function $g_{n}$ (Section 6.2). The case where all eigenvalues are equal to zero gives rise to the theory of П-regular variation of arbitrary order, see Omey and Segers 2009 , sections $5-6,8)$.

1.3. Conventions. A real function is defined in a neighbourhood of infinity if its domain contains an interval of the form $\left[t_{0}, \infty\right)$ for some real $t_{0}$. A real-valued function $f$ defined in a neighbourhood of infinity is eventually of constant sign if there exists a real $t_{0}$ such that either $f(t)>0$ for all $t \geqslant t_{0}$ or $f(t)<0$ for all $t \geqslant t_{0}$. Measure and measurability refer to the Lebesgue measure and $\sigma$-algebra.

Unless specified otherwise, limit relations are to be understood as $t \rightarrow \infty$. We write $a(t) \sim b(t)$ if $a(t) / b(t) \rightarrow 1$.

A column vector $\boldsymbol{g}=\left(g_{1}, \ldots, g_{n}\right)^{\prime}$ of real-valued functions defined in a neighbourhood of infinity is called a rate vector if each component function is ultimately of constant sign and, in case $n \geqslant 2$, if $g_{i+1}(t)=o\left(g_{i}(t)\right)$ for every $i \in\{1, \ldots, n-1\}$. Even though the sign of $g_{i}(t)$ could be eventually negative, for simplicity we will write $o\left(g_{i}(t)\right)$ rather than $o\left(\left|g_{i}(t)\right|\right)$.

2. Quantifiers and limits. For a rate vector $\boldsymbol{g}$ of length $n$, let $S(\boldsymbol{g})$ be the set of all $x \in(0, \infty)$ for which there exists $\boldsymbol{A}(x) \in \mathbb{R}^{n \times n}$ such that

$$
\boldsymbol{g}(x t)=\boldsymbol{A}(x) \boldsymbol{g}(t)+o\left(g_{n}(t)\right) .
$$

For a real-valued function $f$ defined in a neighbourhood of infinity and a rate vector $\boldsymbol{g}$ of length $n$, let $T(f, \boldsymbol{g})$ be the set of all $x \in(0, \infty)$ for which there exists a row vector $\boldsymbol{h}(x)=\left(h_{1}(x), \ldots, h_{n}(x)\right)$ such that

$$
f(x t)=f(t)+\boldsymbol{h}(x) \boldsymbol{g}(t)+o\left(g_{n}(t)\right) .
$$

Whenever clear from the context, we will just write $S=S(\boldsymbol{g})$ and $T=T(f, \boldsymbol{g})$.

In this section, we will study properties of and relations between the sets $S$ and $T$ and the matrix and vector functions $\boldsymbol{A}: S \rightarrow \mathbb{R}^{n \times n}$ and $\boldsymbol{h}: T \rightarrow \mathbb{R}^{1 \times n}$. In particular, we investigate the extent to which (2.1) is implied by (2.2). In $\$ 2.1$. we study the properties of $\boldsymbol{A}$ and $S$, while in $\$ 2.2$ we study the interplay between $\boldsymbol{A}$ and $S$ on the one hand and $\boldsymbol{h}$ and $T$ on the other hand. We do not assume that $f$ or $\boldsymbol{g}$ are measurable; this assumption will be added from the next section onwards. 
REMARK 2.1. Let $f$ be a real-valued function defined in a neighbourhood of infinity and let $\boldsymbol{g}$ be a rate vector.

(a) For $x \in T$, the vector $\boldsymbol{h}(x)$ is uniquely defined by $f$ and $\boldsymbol{g}$ through the recursive relations

$$
\begin{aligned}
& h_{1}(x)=\lim _{t \rightarrow \infty}\{f(t x)-f(t)\} / g_{1}(t), \\
& h_{k}(x)=\lim _{t \rightarrow \infty}\left\{f(t x)-f(t)-\sum_{i=1}^{k-1} h_{i}(x) g_{i}(t)\right\} / g_{k}(t), \quad k \in\{2, \ldots, n\} .
\end{aligned}
$$

(b) If $\boldsymbol{Q}$ is an invertible, upper triangular $n \times n$ matrix, then $\boldsymbol{Q} \boldsymbol{g}$ is a rate vector as well and (2.2) holds true with $\boldsymbol{g}(t)$ and $\boldsymbol{h}(x)$ replaced by $\boldsymbol{Q g}(t)$ and $\boldsymbol{h}(x) \boldsymbol{Q}^{-1}$, respectively.

(c) Rather than 2.2 , one could also study more general relations of the form

$$
f(t x)=x^{\alpha} f(t)+\boldsymbol{h}(x) \boldsymbol{g}(t)+o\left(g_{n}(t)\right)
$$

for some fixed $\alpha \in \mathbb{R}$. However, replacing $f(t)$ by $t^{-\alpha} f(t), \boldsymbol{g}(t)$ by $t^{-\alpha} \boldsymbol{g}(t)$ and $\boldsymbol{h}(x)$ by $x^{-\alpha} \boldsymbol{h}(x)$ would lead back to 2.2 again.

2.1. Properties of $\boldsymbol{A}$ and $S$. We study some elementary properties of the set $S$ and the matrix function $\boldsymbol{A}$ in (2.1). Most importantly, the matrices $\boldsymbol{A}(x)$ are upper triangular, the matrix function $x \mapsto \boldsymbol{A}(x)$ is multiplicative and the set $S$ is a multiplicative semigroup (Proposition 2.2). As a consequence, if $S$ contains a set of positive measure and if both $S \cap(0,1)$ and $S \cap(1, \infty)$ are non-empty, then actually $S=(0, \infty)$ (Proposition 2.3).

Proposition 2.2. Let $\boldsymbol{g}$ be a rate vector and let $S=S(\boldsymbol{g})$ and $\boldsymbol{A}(x)$ be as in 2.1.

(a) The matrix $\boldsymbol{A}(x)$ is upper triangular and is uniquely determined by $\boldsymbol{g}$ and $x$.

(b) If $x, y \in S$ then $x y \in S$ and $\boldsymbol{A}(x y)=\boldsymbol{A}(x) \boldsymbol{A}(y)$.

(c) For $x \in S$, the matrix $\boldsymbol{A}(x)$ is invertible if and only if $x^{-1} \in S$; in that case, $\boldsymbol{A}(x)^{-1}=\boldsymbol{A}\left(x^{-1}\right)$.

Proof. (a) If $n=1$, then 2.1) is just $g_{1}(x t) / g_{1}(t) \rightarrow A_{11}(x)$, so there is nothing to prove. Suppose therefore that $n \geqslant 2$. Row number $i \in\{1, \ldots, n\}$ in 2.1 reads

$$
g_{i}(x t)=\sum_{j=1}^{n} A_{i j}(x) g_{j}(t)+o\left(g_{n}(t)\right), \quad x \in S .
$$

Since $\boldsymbol{g}$ is a rate vector, we find

$$
g_{i}(x t) / g_{1}(t) \rightarrow A_{i 1}(x), \quad x \in S .
$$

If $i \geqslant 2$, then also

$$
\frac{g_{i}(x t)}{g_{1}(t)}=\frac{g_{i}(x t)}{g_{1}(x t)} \frac{g_{1}(x t)}{g_{1}(t)} \rightarrow 0 \cdot A_{11}(x)=0, \quad x \in S .
$$

As a consequence, $A_{i 1}(x)=0$ for $x \in S$ and $i \in\{2, \ldots, n\}$. Therefore, the vector $\left(g_{2}, \ldots, g_{n}\right)^{\prime}$, which is a rate vector too, satisfies 2.1$)$ as well but with $\boldsymbol{A}(x)$ replaced by $\left(A_{i j}(x)\right)_{i, j=2}^{n}, x \in S$. Proceed by induction to find that $\boldsymbol{A}(x)$ is upper triangular. From

$$
g_{i}(x t)=\sum_{j=i}^{n} A_{i j}(x) g_{j}(t)+o\left(g_{n}(t)\right), \quad x \in S, i \in\{1, \ldots, n\}
$$


and the fact that $\boldsymbol{g}$ is a rate vector, it follows that the component functions $A_{i j}$ can be retrieved recursively from $\boldsymbol{g}$ via the relations

$$
\begin{aligned}
& A_{i i}(x)=\lim _{t \rightarrow \infty} g_{i}(x t) / g_{i}(t), \\
& A_{i j}(x)=\lim _{t \rightarrow \infty}\left(g_{i}(x t)-\sum_{k=i}^{j-1} A_{i k}(x) g_{k}(t)\right) / g_{j}(t)
\end{aligned}
$$

for $i \in\{1, \ldots, n\}$ and $j \in\{i+1, \ldots, n\}$.

(b) For $x, y \in S$, we have

$$
\begin{aligned}
\boldsymbol{g}(x y t) & =\boldsymbol{A}(x) \boldsymbol{g}(y t)+o\left(g_{n}(y t)\right) \\
& =\boldsymbol{A}(x) \boldsymbol{A}(y) \boldsymbol{g}(t)+o\left(g_{n}(t)\right)+o\left(g_{n}(y t)\right) .
\end{aligned}
$$

Since $g_{n}(y t) / g_{n}(t) \rightarrow A_{n n}(y)$, we obtain

$$
\boldsymbol{g}(x y t)=\boldsymbol{A}(x) \boldsymbol{A}(y) \boldsymbol{g}(t)+o\left(g_{n}(t)\right),
$$

and thus $x y \in S$. Moreover, $\boldsymbol{A}(x y)=\boldsymbol{A}(x) \boldsymbol{A}(y)$ by uniqueness.

(c) Trivially, $1 \in S$ and $\boldsymbol{A}(1)=I_{n}$, the $n \times n$ identity matrix. Therefore, if both $x$ and $x^{-1}$ belong to $S$, then by (b), $\boldsymbol{A}(x) \boldsymbol{A}\left(x^{-1}\right)=\boldsymbol{A}\left(x x^{-1}\right)=\boldsymbol{A}(1)=I_{n}$. Conversely, suppose that $x \in S$ and that $\boldsymbol{A}(x)$ is invertible. Then

$$
\begin{aligned}
\boldsymbol{A}(x)^{-1} \boldsymbol{g}(x t) & =\boldsymbol{A}(x)^{-1}\left\{\boldsymbol{A}(x) \boldsymbol{g}(t)+o\left(g_{n}(t)\right)\right\} \\
& =\boldsymbol{g}(t)+o\left(g_{n}(t)\right) .
\end{aligned}
$$

From the fact that $\boldsymbol{A}(x)$ is upper triangular and invertible, it follows that $A_{n n}(x)$ is nonzero. But as $g_{n}(x t) / g_{n}(t) \rightarrow A_{n n}(x)$, we may therefore rewrite the previous display as

$$
\boldsymbol{A}(x)^{-1} \boldsymbol{g}(x t)=\boldsymbol{g}\left(x^{-1}(x t)\right)+o\left(g_{n}(x t)\right) .
$$

Putting $s=x t$, we get

$$
\boldsymbol{g}\left(x^{-1} s\right)=\boldsymbol{A}(x)^{-1} \boldsymbol{g}(s)+o\left(g_{n}(s)\right), \quad s \rightarrow \infty .
$$

As a consequence, $x^{-1} \in S$. Moreover, by uniqueness, $\boldsymbol{A}\left(x^{-1}\right)=\boldsymbol{A}(x)^{-1}$.

Proposition 2.3. Let $\boldsymbol{g}$ be a rate vector and let $S=S(\boldsymbol{g})$.

(a) If $S \cap(1, \infty)$ contains a set of positive measure, then $(x, \infty) \subset S$ for some $x \in$ $(1, \infty)$.

(b) If $S \cap(0,1)$ contains a set of positive measure, then $(0, x) \subset S$ for some $x \in(0,1)$.

(c) If $S$ contains a set of positive measure and if both $S \cap(0,1)$ and $S \cap(1, \infty)$ are non-empty, then $S=(0, \infty)$.

Proof. By Proposition 2.2, $S$ is a multiplicative semigroup. Statements (a) and (b) then follow directly from Corollary 1.1.5 in Bingham et al. (1987), due to Hille and Phillips (1974).

To prove (c), proceed as follows. By assumption, $S$ contains a set of positive measure; hence $S \cap(0,1)$ or $S \cap(1, \infty)$ must contain a set of positive measure; assume the latter. Take $y \in S \cap(0,1)$, which is non-empty by assumption. By (a), there exists a positive integer $k$ such that $\left(y^{-k}, \infty\right) \subset S$. Since $y \in S$ and since $S$ is a multiplicative semigroup, 
$(a, \infty) \subset S$ implies $(a y, \infty) \subset S$. By induction, we get that $\left(y^{l}, \infty\right) \subset S$ for every integer l. Hence $(0, \infty) \subset S$.

2.2. Interplay between $\boldsymbol{A}, S$ and $\boldsymbol{h}, T$. Next we study the interplay between $\boldsymbol{A}$ and $S$ for $\boldsymbol{g}$ in (2.1) on the one hand and $\boldsymbol{h}$ and $T$ for $f$ in 2.2 on the other hand. In Proposition 2.4 we describe how properties of $S$ transfer to properties of $T$ and we derive some functional relations between $\boldsymbol{A}$ and $\boldsymbol{h}$. Conversely, in Proposition 2.6, we investigate the extent to which 2.2 implies 2.1); in particular, we express $\boldsymbol{g}$ and $\boldsymbol{A}$ in terms of $f$ and $\boldsymbol{h}$.

Proposition 2.4. Let $f$ be a real-valued function defined in a neighbourhood of infinity and let $\boldsymbol{g}$ be a rate vector. If $S=(0, \infty)$, then $T$ is a multiplicative group and for $x, y \in T$,

$$
\begin{aligned}
\boldsymbol{h}(x y) & =\boldsymbol{h}(x) \boldsymbol{A}(y)+\boldsymbol{h}(y), \\
\boldsymbol{h}\left(x^{-1}\right) & =-\boldsymbol{h}(x) \boldsymbol{A}(x)^{-1} .
\end{aligned}
$$

In particular, if $T$ also contains a set of positive measure, then $T=(0, \infty)$.

Proof. Let $x, y \in T$. We have

$$
\begin{aligned}
f(x y t)-f(t) & =\{f(x y t)-f(y t)\}+\{f(y t)-f(t)\} \\
& =\boldsymbol{h}(x) \boldsymbol{g}(y t)+o\left(g_{n}(y t)\right)+\boldsymbol{h}(y) \boldsymbol{g}(t)+o\left(g_{n}(t)\right) \\
& =\boldsymbol{h}(x) \boldsymbol{A}(y) \boldsymbol{g}(t)+\boldsymbol{h}(y) \boldsymbol{g}(t)+o\left(g_{n}(y t)\right)+o\left(g_{n}(t)\right) \\
& =\{\boldsymbol{h}(x) \boldsymbol{A}(y)+\boldsymbol{h}(y)\} \boldsymbol{g}(t)+o\left(g_{n}(t)\right),
\end{aligned}
$$

where in the last step we used the fact that $g_{n}(y t) / g_{n}(t) \rightarrow A_{n n}(y)$. Hence $x y \in T$, and (2.5) follows from the uniqueness of $\boldsymbol{h}$.

Next, let $x \in T$. We have

$$
\begin{aligned}
f\left(x^{-1} t\right)-f(t) & =-\left\{f\left(x\left(x^{-1} t\right)\right)-f\left(x^{-1} t\right)\right\} \\
& =-\boldsymbol{h}(x) \boldsymbol{g}\left(x^{-1} t\right)+o\left(g_{n}\left(x^{-1} t\right)\right) \\
& =-\boldsymbol{h}(x) \boldsymbol{A}\left(x^{-1}\right) \boldsymbol{g}(t)+o\left(g_{n}(t)\right)+o\left(g_{n}\left(x^{-1} t\right)\right) \\
& =-\boldsymbol{h}(x) \boldsymbol{A}\left(x^{-1}\right) \boldsymbol{g}(t)+o\left(g_{n}(t)\right),
\end{aligned}
$$

where in the last step we used the fact that $g_{n}\left(x^{-1} t\right) / g_{n}(t) \rightarrow A_{n n}\left(x^{-1}\right)$. Hence $x^{-1} \in T$, and 2.6) follows from the uniqueness of $\boldsymbol{h}$.

The final statement follows Bingham et al. (1987, Corollary 1.1.4), going back to Steinhaus (1920).

REMARK 2.5. Let $f$ be a real-valued function defined in a neighbourhood of infinity and let $\boldsymbol{g}=\left(g_{1}, \ldots, g_{n}\right)^{\prime}$ be a rate vector. Put

$$
\begin{aligned}
\boldsymbol{r} & =\left(f, g_{1}, \ldots, g_{n}\right)^{\prime}, \\
\boldsymbol{C}(x) & =\left(\begin{array}{cc}
1 & \boldsymbol{h}(x) \\
\mathbf{0} & \boldsymbol{A}(x)
\end{array}\right), \quad x \in S \cap T .
\end{aligned}
$$

By Proposition 2.2(a), $\boldsymbol{C}(x)$ is an $(n+1) \times(n+1)$ upper triangular matrix. Then 2.1 and 2.2 can be put together as

$$
\boldsymbol{r}(x t)=\boldsymbol{C}(x) \boldsymbol{r}(t)+o\left(r_{n+1}(t)\right),
$$


which is again of the form 2.1). If $x, y \in S \cap T$, then by going through the proof of Proposition 2.4 we find that also $x y \in S \cap T$ and that $\boldsymbol{C}(x y)=\boldsymbol{C}(x) \boldsymbol{C}(y)$. Similarly, for $x \in S \cap T$, the matrix $\boldsymbol{C}(x)$ is invertible if and only if $x^{-1} \in S \cap T$, in which case $\boldsymbol{C}\left(x^{-1}\right)=\boldsymbol{C}(x)^{-1}$.

However, $\boldsymbol{r}$ is not necessarily a rate vector. Moreover, as we shall be interested in the interplay between (2.1) and (2.2), we continue to study these equations separately.

Conversely, to go from $T$ and $\boldsymbol{h}$ to $S$ and $\boldsymbol{A}$, we need to impose that the functions $h_{1}, \ldots, h_{n}$ are linearly independent. For instance, if $n=1$ and $h_{1} \equiv 0$, then 2.2 reduces to $f(x t)-f(t)=o(g(t))$, from which in general nothing can be deduced about the rate function $g$; likewise, in case $n=2$, linear independence of the functions $h_{1}$ and $h_{2}$ is assumed from the start in de Haan and Stadtmüller (1996). Note that by Remark 2.7 below, unless the functions $h_{1}, \ldots, h_{n}$ are all identically zero, it is always possible to find a non-empty $I \subset\{1, \ldots, n\}$ and a rate vector $\tilde{\boldsymbol{g}}=\left(\tilde{g}_{i}\right)_{i \in I}^{\prime}$ such that the functions $h_{i}, i \in I$ are linearly independent, $\tilde{g}_{i}(t) / g_{i}(t) \rightarrow 1$ for all $i \in I$, and $f(x t)=f(t)+$ $\sum_{i \in I} h_{i}(x) \tilde{g}_{i}(t)+o\left(g_{n}(t)\right)$.

Notation. Let $f$ and $\boldsymbol{h}$ be as in 2.2. For a vector $\boldsymbol{x}=\left(x_{1}, \ldots, x_{n}\right) \in T^{n}$, put

$$
\boldsymbol{h}(\boldsymbol{x})=\left(\boldsymbol{h}\left(x_{1}\right)^{\prime}, \ldots, \boldsymbol{h}\left(x_{n}\right)^{\prime}\right)^{\prime},
$$

that is, $\boldsymbol{h}(\boldsymbol{x})$ is an $n \times n$-matrix with $\boldsymbol{h}\left(x_{i}\right)$ as row number $i$. Further, for $\boldsymbol{t}=\left(t_{1}, \ldots, t_{n}\right)$ such that all $t_{i}$ are in the domain of $f$, put

$$
f(\boldsymbol{t})=\left(f\left(t_{1}\right), \ldots, f\left(t_{n}\right)\right)^{\prime} .
$$

Finally, put $\mathbf{1}=(1, \ldots, 1) \in \mathbb{R}^{n}$.

Note that the functions $h_{1}, \ldots, h_{n}$ are linearly independent if and only if there exists $\boldsymbol{x} \in T^{n}$ such that the vectors $\left(h_{i}\left(x_{1}\right), \ldots, h_{i}\left(x_{n}\right)\right), i \in\{1, \ldots, n\}$ are independent (Cheney and Light, 2000, Chapter 1, Problem 8), i.e., the matrix $\boldsymbol{h}(\boldsymbol{x})$ is invertible.

Proposition 2.6. Let $f$ be a real-valued function defined in a neighbourhood of infinity and let $\boldsymbol{g}$ be a rate vector. If $T=(0, \infty)$ and if the functions $h_{1}, \ldots, h_{n}$ in (2.2) are linearly independent, then $S=(0, \infty)$, and for $y \in(0, \infty)$ and for any $\boldsymbol{x} \in(0, \infty)^{n}$ such that $\boldsymbol{h}(\boldsymbol{x})$ is invertible,

$$
\begin{aligned}
\boldsymbol{g}(t) & =\boldsymbol{h}(\boldsymbol{x})^{-1}\{f(t \boldsymbol{x})-f(t \mathbf{1})\}+o\left(g_{n}(t)\right), \\
\boldsymbol{A}(y) & =\boldsymbol{h}(\boldsymbol{x})^{-1}\{\boldsymbol{h}(y \boldsymbol{x})-\boldsymbol{h}(y \mathbf{1})\} .
\end{aligned}
$$

Proof. For $\boldsymbol{x} \in(0, \infty)^{n}$ and $i \in\{1, \ldots, n\}$, we have

$$
f\left(x_{i} t\right)=f(t)+\boldsymbol{h}\left(x_{i}\right) \boldsymbol{g}(t)+o\left(g_{n}(t)\right) ;
$$

in matrix notation, this becomes

$$
f(t \boldsymbol{x})=f(t \mathbf{1})+\boldsymbol{h}(\boldsymbol{x}) \boldsymbol{g}(t)+o\left(g_{n}(t)\right) .
$$

Now let $\boldsymbol{x} \in(0, \infty)^{n}$ be such that $\boldsymbol{h}(\boldsymbol{x})$ is invertible. Then (2.11) clearly implies 2.9 . 
For $y \in(0, \infty)$, we have by 2.9 and 2.11

$$
\begin{aligned}
\boldsymbol{g}(t y) & =\boldsymbol{h}(\boldsymbol{x})^{-1}\{f(t y \boldsymbol{x})-f(t y \mathbf{1})\}+o\left(g_{n}(y t)\right) \\
& =\boldsymbol{h}(\boldsymbol{x})^{-1}\{f(t y \boldsymbol{x})-f(t \mathbf{1})\}-\boldsymbol{h}(\boldsymbol{x})^{-1}\{f(t y \mathbf{1})-f(t \mathbf{1})\}+o\left(g_{n}(y t)\right) \\
& =\boldsymbol{h}(\boldsymbol{x})^{-1} \boldsymbol{h}(y \boldsymbol{x}) \boldsymbol{g}(t)-\boldsymbol{h}(\boldsymbol{x})^{-1} \boldsymbol{h}(y \mathbf{1}) \boldsymbol{g}(t)+o\left(g_{n}(t)\right)+o\left(g_{n}(y t)\right) \\
& =\boldsymbol{A}(y) \boldsymbol{g}(t)+o\left(g_{n}(t)\right)+o\left(g_{n}(y t)\right),
\end{aligned}
$$

with $\boldsymbol{A}(y)=\boldsymbol{h}(\boldsymbol{x})^{-1}\{\boldsymbol{h}(y \boldsymbol{x})-\boldsymbol{h}(y \mathbf{1})\}$. Row $i$ of 2.12 reads

$$
g_{i}(t y)=\sum_{j=1}^{n} A_{i j}(y) g_{j}(t)+o\left(g_{n}(t)\right)+o\left(g_{n}(t y)\right) .
$$

In particular, $g_{1}(t y)=A_{11}(y) g_{1}(t)+o\left(g_{1}(t)\right)+o\left(g_{1}(t y)\right)$ and thus

$$
g_{1}(t y) / g_{1}(t) \rightarrow A_{11}(y) .
$$

For $i \in\{2, \ldots, n\}$, we find from the two previous displays that

$$
A_{i 1}(y)=\lim _{t \rightarrow \infty} g_{i}(t y) / g_{1}(t)=0,
$$

and thus

$$
g_{i}(t y)=\sum_{j=2}^{n} A_{i j}(y) g_{j}(t)+o\left(g_{n}(t)\right)+o\left(g_{n}(t y)\right), \quad i \in\{2, \ldots, n\} .
$$

Repeating the same argument inductively yields eventually

$$
g_{n}(t y)=A_{n n}(y) g_{n}(y)+o\left(g_{n}(t)\right)+o\left(g_{n}(t y)\right)
$$

from which

$$
g_{n}(t y) / g_{n}(t) \rightarrow A_{n n}(y) .
$$

Hence, on the right-hand side in (2.12), the term $o\left(g_{n}(t y)\right)$ can be absorbed by the term $o\left(g_{n}(t)\right)$, yielding $\boldsymbol{g}(t y)=\boldsymbol{A}(y) \boldsymbol{g}(t)+o\left(g_{n}(t)\right)$. Hence $y \in S$ with $\boldsymbol{A}(y)$ as in 2.10.

REMARK 2.7. Let $f$ be a real-valued function defined in a neighbourhood of infinity, let $\boldsymbol{g}$ be a rate vector, and assume that not all functions $h_{1}, \ldots, h_{n}$ in $(2.2)$ are identically zero. Then by basic linear algebra, there exists a non-empty subset $I$ of $\{1, \ldots, n\}$ such that the following holds:

(i) the functions $h_{i}, i \in I$, are linearly independent;

(ii) there exists an array of real numbers $\lambda_{i j}$, where $i \in\{1, \ldots, n\}$ and $j \in I \cap\{1, \ldots, i\}$, such that $h_{i}=\sum_{j \in I, j \leqslant i} \lambda_{i j} h_{j}$ (the empty sum being zero by convention).

Then

$$
\boldsymbol{h}(x) \boldsymbol{g}(t)=\sum_{i=1}^{n} \sum_{j \in I, j \leqslant i} \lambda_{i j} h_{j}(x) g_{i}(t)=\sum_{j \in I} h_{j}(x) \tilde{g}_{j}(t)
$$

with $\tilde{g}_{j}(t)=\sum_{i=j}^{n} \lambda_{i j} g_{i}(t)$. Since $\lambda_{j j}=1$ for $j \in I$, we have $\tilde{g}_{j}(t) / g_{j}(t) \rightarrow 1$; in particular, $\left(\tilde{g}_{j}\right)_{j \in I}$ is a rate vector.

REMARK 2.8. Let $f$ be a real-valued function defined in a neighbourhood of infinity, let $\boldsymbol{g}$ be a rate vector, and assume that there exists $\boldsymbol{x} \in T^{n}$ such that $\boldsymbol{h}(\boldsymbol{x})$ is invertible. Let

$$
T(\boldsymbol{x})=\left\{y \in T: x_{i} y \in T, \forall i\right\} .
$$


By repeating the proof of Proposition 2.6. we see that $T(\boldsymbol{x}) \subset S$. As a consequence, if $T(\boldsymbol{x})$ contains a set of positive measure and if both $T(\boldsymbol{x}) \cap(0,1)$ and $T(\boldsymbol{x}) \cap(1, \infty)$ are non-empty, then by Proposition 2.3(c) and Proposition 2.4 actually $S=(0, \infty)$ and $T=(0, \infty)$.

3. Regularly varying rate vectors and generalized regular variation. Up to now, we have studied the asymptotic relations (2.1) and (2.2) without assuming that $f$ or $\boldsymbol{g}$ are measurable. This assumption is added now. In addition, we require that $S=(0, \infty)$ and $T=(0, \infty)$. In that case, the limit functions $\boldsymbol{A}$ and $\boldsymbol{h}$ admit particularly simple analytic forms in terms of matrix exponential functions. Some preliminaries on matrix exponentials are collected in Appendix A. In 3.1. we focus on the rate vector $\boldsymbol{g}$, whereas in 3.2 , we turn to the function $f$ itself.

3.1. Regularly varying rate vectors. For measurable rate vectors $\boldsymbol{g}$ satisfying (3.1) for all $x \in(0, \infty)$, the matrix function $\boldsymbol{A}$ can be written as $\boldsymbol{A}(x)=x^{\boldsymbol{B}}$ for some upper triangular matrix $\boldsymbol{B}$, the diagonal elements of $\boldsymbol{B}$ being the indices of regular variation of the component functions $\left|g_{i}\right|$ (Propositions 3.1 and 3.2. This motivates the definition of the class $R V_{\boldsymbol{B}}$ of regularly varying rate vectors with index matrix $\boldsymbol{B}$. The requirement that the convergence holds for all $x \in(0, \infty)$ can be relaxed considerably (Theorem 3.4).

Proposition 3.1. Let $\boldsymbol{g}$ be a measurable rate vector. If there exists $\boldsymbol{A}:(0, \infty) \rightarrow \mathbb{R}^{n \times n}$ such that

$$
\boldsymbol{g}(x t)=\boldsymbol{A}(x) \boldsymbol{g}(t)+o\left(g_{n}(t)\right), \quad x \in(0, \infty),
$$

then there exists an upper triangular matrix $\boldsymbol{B} \in \mathbb{R}^{n \times n}$ such that

$$
\boldsymbol{A}(x)=x^{\boldsymbol{B}}, \quad x \in(0, \infty) .
$$

Proof. From (2.3)-(2.4) it follows that $\boldsymbol{A}$ is measurable. Hence, in view of Proposition 2.2 $\boldsymbol{A}$ is a measurable group homomorphism from $(0, \infty)$ into the group of invertible $n \times n$ matrices. Then necessarily $\boldsymbol{A}(x)=x^{\boldsymbol{B}}, x \in(0, \infty)$, with

$$
\boldsymbol{B}=\lim _{x \rightarrow 1} \frac{\boldsymbol{A}(x)-\boldsymbol{I}}{x-1}
$$

see for instance Dunford and Schwartz (1958, Theorem VIII.1.2 and Lemma VIII.1.3). From the above display, it follows that $\boldsymbol{B}$ is upper triangular.

Proposition 3.2. Let $\boldsymbol{B}$ be the matrix appearing in Proposition 3.1 and put $b_{i}=B_{i i}$, $i \in\{1, \ldots, n\}$.

(a) The function $\left|g_{i}\right|$ is regularly varying with index $b_{i}$; in particular, $b_{1} \geqslant \cdots \geqslant b_{n}$.

(b) All the eigenvalues of $\boldsymbol{B}$, except maybe for the smallest one, have geometric multiplicity equal to one.

Proof. (a) This follows from the fact that

$$
g_{i}(x t) / g_{i}(t) \rightarrow A_{i i}(x)=x^{b_{i}}, \quad x \in(0, \infty), \quad i \in\{1, \ldots, n\} .
$$

Since $g_{i}(t)=o\left(g_{i-1}(t)\right)$, necessarily $b_{i} \leqslant b_{i-1}$. 
(b) Let $b$ be an eigenvalue (diagonal element) of $\boldsymbol{B}$ and let $\boldsymbol{v} \in \mathbb{R}^{1 \times n}$ satisfy $\boldsymbol{v} \boldsymbol{B}=b \boldsymbol{v}$. Then $\boldsymbol{v} x^{\boldsymbol{B}}=x^{b} \boldsymbol{v}$ for all $x>0$. Hence

$$
\boldsymbol{v} \boldsymbol{g}(x t)=\boldsymbol{v} x^{\boldsymbol{B}} \boldsymbol{g}(t)+o\left(g_{n}(t)\right)=x^{b} \boldsymbol{v} \boldsymbol{g}(t)+o\left(g_{n}(t)\right), \quad x \in(0, \infty),
$$

and thus

$$
(x t)^{-b} \boldsymbol{v g}(x t)=t^{-b} \boldsymbol{v} \boldsymbol{g}(t)+o\left(t^{-b} g_{n}(t)\right), \quad x \in(0, \infty) .
$$

This states that the function $t \mapsto t^{-b} \boldsymbol{v g}(t)$ belongs to the class $o \Pi_{a}$ with $a(t)=t^{-b} g_{n}(t)$. Now unless $b$ is equal to the smallest eigenvalue, $b_{n}$, the Representation Theorem for $o \Pi$ (Bingham et al., 1987, Theorem 3.6.1) stipulates the existence of $c \in \mathbb{R}$ such that

$$
t^{-b} \boldsymbol{v g}(t)=c+o\left(t^{-b} g_{n}(t)\right),
$$

or in other words

$$
\boldsymbol{v} \boldsymbol{g}(t)=c t^{b}+o\left(g_{n}(t)\right) \text {. }
$$

Now let $b>b_{n}$ and suppose that $\boldsymbol{v}_{1}, \boldsymbol{v}_{2} \in \mathbb{R}^{1 \times n}$ both satisfy $\boldsymbol{v}_{i} \boldsymbol{B}=b \boldsymbol{v}_{i}$, for $i=1$, Let $c_{1}, c_{2} \in \mathbb{R}$ be such that $\boldsymbol{v}_{i} \boldsymbol{g}(t)=c_{i} t^{b}+o\left(g_{n}(t)\right)$, for $i=1,2$. Find real numbers $\lambda_{1}$ and $\lambda_{2}$, not both zero, such that $\lambda_{1} c_{1}+\lambda_{2} c_{2}=0$. The vector $\boldsymbol{v}=\lambda_{1} \boldsymbol{v}_{1}+\lambda_{2} \boldsymbol{v}_{2}$ clearly satisfies $\boldsymbol{v} \boldsymbol{g}(t)=o\left(g_{n}(t)\right)$. As $\boldsymbol{g}$ is a rate vector, necessarily $\boldsymbol{v}=\mathbf{0}$. Hence $\boldsymbol{v}_{1}$ and $\boldsymbol{v}_{2}$ are linearly dependent.

These results motivate the following definition.

Definition 3.3. A matrix $\boldsymbol{B} \in \mathbb{R}^{n \times n}$ is an index matrix if it is upper triangular, if its diagonal elements are non-increasing, and if all of its eigenvalues, except maybe for the smallest one, have geometric multiplicity equal to one.

A rate vector $\boldsymbol{g}$ of length $n$ is regularly varying with index matrix $\boldsymbol{B}$ if $\boldsymbol{g}$ is measurable and

$$
\boldsymbol{g}(x t)=x^{\boldsymbol{B}} \boldsymbol{g}(t)+o\left(g_{n}(t)\right), \quad x \in(0, \infty)
$$

Notation: $\boldsymbol{g} \in R V_{\boldsymbol{B}}$.

Combining Propositions 2.3. (c), 3.1, and 3.2, we arrive at our first main result.

THEOREM 3.4 (Characterization Theorem for $R V_{\boldsymbol{B}}$ ). Let $\boldsymbol{g}$ be a measurable rate vector of length $n$ and let $S$ be the set of $x \in(0, \infty)$ for which there exists $\boldsymbol{A}(x) \in \mathbb{R}^{n \times n}$ such that $\boldsymbol{g}(x t)=\boldsymbol{A}(x) \boldsymbol{g}(t)+o\left(g_{n}(t)\right)$. If $S$ contains a set of positive measure and if both $S \cap(0,1)$ and $S \cap(1, \infty)$ are non-empty, then $\boldsymbol{g} \in R V_{\boldsymbol{B}}$ for some index matrix $\boldsymbol{B}$.

REMARK 3.5. Let $\boldsymbol{g} \in R V_{\boldsymbol{B}}$.

(a) If $\boldsymbol{Q} \in \mathbb{R}^{n \times n}$ is upper triangular and invertible, then $\boldsymbol{Q g}$ is also a rate vector and $\boldsymbol{Q g} \in R V_{\boldsymbol{Q B} \boldsymbol{Q}^{-1}}$.

(b) For integer $1 \leqslant k \leqslant l \leqslant n$, the subvector $\boldsymbol{g}_{k l}=\left(g_{k}, \ldots, g_{l}\right)^{\prime}$ is also a rate vector and $\boldsymbol{g}_{k l} \in R V_{\boldsymbol{B}_{k l}}$, where $\boldsymbol{B}_{k l}=\left(B_{i j}\right)_{i, j=k}^{l}$.

3.2. Generalized regular variation. Next we resume the study of the relation $f(x t)=$ $f(t)+\boldsymbol{h}(x) \boldsymbol{g}(t)+o\left(g_{n}(t)\right)$, this time for measurable $f$. In view of Proposition 2.6, there is not much harm in assuming from the start that $\boldsymbol{g}$ is measurable as well. 
Definition 3.6. A measurable, real-valued function $f$ defined in a neighbourhood of infinity is generalized regularly varying with rate vector $\boldsymbol{g} \in R V_{\boldsymbol{B}}$ and $\boldsymbol{g}$-index $\boldsymbol{c} \in \mathbb{R}^{1 \times n}$ if

$$
f(x t)=f(t)+\boldsymbol{h}(x) \boldsymbol{g}(t)+o\left(g_{n}(t)\right), \quad x \in(0, \infty),
$$

where

$$
\boldsymbol{h}(x)=\boldsymbol{c} \int_{1}^{x} y^{\boldsymbol{B}} y^{-1} \mathrm{~d} y, \quad x \in(0, \infty) .
$$

Notation: $f \in G R V(\boldsymbol{g})$.

Our second main result asserts that in the relation $(3.2)$, the regular variation property of $\boldsymbol{g}$ and the form of the limit function $\boldsymbol{h}$ in (3.3) are automatic. The assumption in Theorem 3.7 that the limit functions $h_{1}, \ldots, h_{n}$ are linearly independent is unavoidable; however, in view of Remark 2.7, unless all $h_{i}$ are identically zero, it is always possible to switch to a subvector $\left(h_{i}\right)_{i \in I}$ of functions that are linearly independent.

Theorem 3.7 (Characterization Theorem for $G R V(\boldsymbol{g})$ ). Let $f$ be a measurable, realvalued function defined in a neighbourhood of infinity and let $\boldsymbol{g}$ be a measurable rate vector of length $n$. Let $T$ be the set of $x \in(0, \infty)$ for which there exists $\boldsymbol{h}(x) \in \mathbb{R}^{1 \times n}$ such that $f(t x)=f(t)+\boldsymbol{h}(x) \boldsymbol{g}(t)+o\left(g_{n}(t)\right)$. Assume that there exists $\boldsymbol{x} \in T^{n}$ such that

(i) the matrix $\boldsymbol{h}(\boldsymbol{x})$ in 2.8 is invertible;

(ii) the set $T(\boldsymbol{x})$ in 2.13 contains a set of positive measure and has non-empty intersections with both $(0,1)$ and $(1, \infty)$.

Then $\boldsymbol{g} \in R V_{\boldsymbol{B}}$ and $f \in G R V(\boldsymbol{g})$ with $\boldsymbol{h}$ as in 3.3 . In addition, all eigenvalues (including the smallest one) of the index matrix $\boldsymbol{B}$ have geometric multiplicity equal to one.

Proof. By Remark 2.8, necessarily $T=(0, \infty)$ and there exists $\boldsymbol{A}:(0, \infty) \rightarrow \mathbb{R}^{n \times n}$ such that $\boldsymbol{g}(t x)=\boldsymbol{A}(x) \boldsymbol{g}(t)+o\left(g_{n}(t)\right)$ for all $x \in(0, \infty)$. By Theorem 3.4, $\boldsymbol{g} \in R V_{\boldsymbol{B}}$ for some index matrix $\boldsymbol{B} \in \mathbb{R}^{n \times n}$.

By Remark 2.5 the matrix function $\boldsymbol{C}:(0, \infty) \rightarrow \mathbb{R}^{(n+1) \times(n+1)}$ in $(2.7)$ is a measurable group homomorphism. As in the proof of Proposition 3.1, we find that $\boldsymbol{C}(x)=x^{\boldsymbol{D}}$ for some upper triangular matrix $\boldsymbol{D} \in \mathbb{R}^{(n+1) \times(n+1)}$. Since $C_{11}(x)=1$ and $\left(C_{i j}(x)\right)_{i, j=2}^{n+1}=$ $\boldsymbol{A}(x)=x^{\boldsymbol{B}}$, the expression for $h$ in $(3.3$ follows from Remark A.2.

Let $b$ be an eigenvalue (i.e. a diagonal element) of $\boldsymbol{B}$ and let $\boldsymbol{v} \in \mathbb{R}^{n \times 1}$ be such that $\boldsymbol{B} \boldsymbol{v}=b \boldsymbol{v}$. For $y \in(0, \infty)$, we have $y^{\boldsymbol{B}} \boldsymbol{v}=y^{b} \boldsymbol{v}$. As a consequence, for $x \in(0, \infty)$,

$$
\boldsymbol{h}(x) \boldsymbol{v}=\boldsymbol{c} \int_{1}^{x} y^{\boldsymbol{B}} y^{-1} \mathrm{~d} y \boldsymbol{v}=\int_{1}^{x} y^{b-1} \mathrm{~d} y \boldsymbol{c} \boldsymbol{v} .
$$

Now let both $\boldsymbol{v}_{1}, \boldsymbol{v}_{2} \in \mathbb{R}^{n \times 1}$ be eigenvectors of $\boldsymbol{B}$ with the same eigenvalue $b$. There exist $\lambda_{1}, \lambda_{2} \in \mathbb{R}$, not both zero, such that $\lambda_{1} \boldsymbol{c} \boldsymbol{v}_{1}+\lambda_{2} \boldsymbol{c} \boldsymbol{v}_{2}=0$, and thus

$$
\boldsymbol{h}(x)\left(\lambda_{1} \boldsymbol{v}_{1}+\lambda_{2} \boldsymbol{v}_{2}\right)=\int_{1}^{x} y^{b-1} \mathrm{~d} y\left(\lambda_{1} \boldsymbol{c} \boldsymbol{v}_{1}+\lambda_{2} \boldsymbol{c} \boldsymbol{v}_{2}\right)=0, \quad x \in(0, \infty) .
$$

Since the functions $h_{1}, \ldots, h_{n}$ were assumed to be linearly independent, the above identity implies that $\lambda_{1} \boldsymbol{v}_{1}+\lambda_{2} \boldsymbol{v}_{2}=\mathbf{0}$, that is, $\boldsymbol{v}_{1}$ and $\boldsymbol{v}_{2}$ are linearly dependent. As a consequence, the dimension of the eigenspace of $b$ cannot be larger than one. 
REMARK 3.8. Let $f \in G R V(\boldsymbol{g})$ with $\boldsymbol{g}$-index $\boldsymbol{c} \in \mathbb{R}^{1 \times n}$. If $\boldsymbol{Q} \in \mathbb{R}^{n \times n}$ is upper triangular and invertible, then also $f \in G R V(\boldsymbol{Q g})$ with $\boldsymbol{Q} \boldsymbol{g}$-index $\boldsymbol{c} \boldsymbol{Q}^{-1}$.

4. Uniform convergence and representations. The asymptotic relations 3.1 and (3.2) defining the function classes $R V_{\boldsymbol{B}}$ and $G R V(\boldsymbol{g})$ respectively hold locally uniformly in $x \in(0, \infty)$, see Theorems 4.1 and 4.2 . These uniform convergence theorems yield representations of $\boldsymbol{g} \in R V_{\boldsymbol{B}}$ and $f \in G R V(\boldsymbol{g})$ in terms of integrals of simpler quantities, see Theorems 4.3 and 4.5 .

THEOREM 4.1 (Uniform convergence theorem for $R V_{\boldsymbol{B}}$ ). If $\boldsymbol{g} \in R V_{\boldsymbol{B}}$, then $\boldsymbol{g}(t x)=$ $x^{B} \boldsymbol{g}(t)+o\left(g_{n}(t)\right)$ locally uniformly in $x \in(0, \infty)$.

Proof. Our proof of Theorem 4.1 is inspired by the one in Delange (1955) for the uniform convergence theorem for slowly varying functions as presented in Bingham et al. (1987, Theorem 1.2.1).

We will prove uniform convergence for $x \in\left[a^{-1}, a\right]$ where $a \in(1, \infty)$. For $\boldsymbol{v}=$ $\left(v_{1}, v_{2}, \ldots, v_{n}\right)$ let $\|\boldsymbol{v}\|=\max \left(\left|v_{1}\right|, \ldots,\left|v_{n}\right|\right)$. The corresponding operator norm on $\mathbb{R}^{n \times n}$ will also be denoted by $\|\cdot\|$. Put $b=B_{n n}$ and choose $\varepsilon \in(0, \log a)$. For $t>0$ sufficiently large, we define the sets

$$
\begin{aligned}
& E(t)=\left\{s \in\left[a^{-1} t, a t\right]:\left\|\boldsymbol{g}(s)-(s / t)^{B} \boldsymbol{g}(t)\right\| \geqslant \varepsilon\left|g_{n}(t)\right|\right\}, \\
& V(t)=\left\{x \in\left[a^{-1}, a\right]:\left\|\boldsymbol{g}(t x)-x^{B} \boldsymbol{g}(t)\right\| \geqslant \varepsilon\left|g_{n}(t)\right|\right\} .
\end{aligned}
$$

These sets are measurable and $E(t)=\{t x: x \in V(t)\}=t V(t)$. With $\mu$ the measure on $(0, \infty)$ defined by $\mu(\mathrm{d} y)=y^{-1} \mathrm{~d} y$, we have $\mu(E(t))=\mu(V(t))$. As $\boldsymbol{g} \in R V_{\boldsymbol{B}}$, it follows that the indicator function of $V(t)$ converges pointwise to zero. By dominated convergence, we have $\mu(V(t)) \rightarrow 0$ and we can find $t_{0}>0$ such that $\mu(E(t))=\mu(V(t)) \leqslant \varepsilon / 2$ for all $t \geqslant t_{0}$.

For $a^{-1} \leqslant x \leqslant a$, the intersection $\left[a^{-1} x t, a x t\right] \cap\left[a^{-1} t, a t\right]$ contains at least one of the intervals $\left[a^{-1} t, t\right]$ or $[t, a t]$. This implies that $\mu\left(\left[a^{-1} x t, a x t\right] \cap\left[a^{-1} t, a t\right]\right) \geqslant \log a$. On the other hand, if $t \geqslant a t_{0}=t_{1}$ and $x \geqslant a^{-1}$, then $\mu(E(t x) \cup E(t)) \leqslant \varepsilon$. Now as $\varepsilon<\log a$, for all $x \in\left[a^{-1}, a\right]$ and $t \geqslant t_{1}$, the set

$$
V(x, t)=\left(\left[a^{-1} x t, a x t\right] \cap\left[a^{-1} t, a t\right]\right) \backslash(E(x t) \cup E(t))
$$

has positive $\mu$-measure and so is certainly non-empty. Let $s=s(x, t) \in V(x, t)$. By definition of $E(t)$ we have for $x \in\left[a^{-1}, a\right]$ and $t \geqslant t_{1}$,

$$
\begin{gathered}
\left\|\boldsymbol{g}(s)-(s / t)^{\boldsymbol{B}} \boldsymbol{g}(t)\right\|<\varepsilon\left|g_{n}(t)\right|, \\
\left\|\boldsymbol{g}(s)-(s /(x t))^{\boldsymbol{B}} \boldsymbol{g}(x t)\right\|<\varepsilon\left|g_{n}(x t)\right|,
\end{gathered}
$$

whence, by the triangle inequality,

$$
\left\|(s /(x t))^{\boldsymbol{B}}\left\{\boldsymbol{x}^{\boldsymbol{B}} \boldsymbol{g}(t)-\boldsymbol{g}(x t)\right\}\right\|<2 \varepsilon\left\{\left|g_{n}(t)\right|+\left|g_{n}(x t)\right|\right\} .
$$

Since the function $\left|g_{n}\right|$ is regularly varying with index $b$, by the Uniform convergence theorem for regularly varying functions (Bingham et al., 1987, Theorem 1.2.1), there exists $t_{2} \geqslant t_{1}$ such that

$$
\left|g_{n}(t x)\right| \leqslant 2 a^{b}\left|g_{n}(t)\right|, \quad x \in\left[a^{-1}, a\right], \quad t \geqslant t_{2} .
$$


Combine the last two displays to see that

$$
\left\|(s /(x t))^{\boldsymbol{B}}\left\{\boldsymbol{x}^{\boldsymbol{B}} \boldsymbol{g}(t)-\boldsymbol{g}(x t)\right\}\right\|<2 \varepsilon\left(1+a^{b}\right)\left|g_{n}(t)\right|, \quad x \in\left[a^{-1}, a\right], \quad t \geqslant t_{2} .
$$

Applying the inequality $\|\boldsymbol{T} \boldsymbol{v}\| \geqslant\left\|\boldsymbol{T}^{-1}\right\|^{-1}\|\boldsymbol{v}\|$, valid for invertible $\boldsymbol{T} \in \mathbb{R}^{n \times n}$, we get from the previous display that

$$
\left\|x^{B} \boldsymbol{g}(t)-\boldsymbol{g}(t x)\right\| \leqslant 2 \varepsilon\left(1+a^{b}\right)\left|g_{n}(t)\right|\left\|(x t / s)^{\boldsymbol{B}}\right\|, \quad x \in\left[a^{-1}, a\right], \quad t \geqslant t_{2} .
$$

Now note that for such $x$ and $t$ we have $a^{-2} \leqslant x t / s \leqslant a^{2}$. Since the function $0<y \mapsto y^{B}$ is continuous, there is a positive constant $C=C(a, \boldsymbol{B})$ such that

$$
\left\|x^{B} \boldsymbol{g}(t)-\boldsymbol{g}(t x)\right\| \leqslant \varepsilon C\left|g_{n}(t)\right|, \quad x \in\left[a^{-1}, a\right], \quad t \geqslant t_{2} .
$$

This proves the result.

THEOREM 4.2 (Uniform convergence theorem for $G R V(\boldsymbol{g})$ ). If $f \in G R V(\boldsymbol{g})$, then the relation $f(t x)=f(t)+\boldsymbol{h}(x) \boldsymbol{g}(t)+o\left(g_{n}(t)\right)$ holds locally uniformly in $x \in(0, \infty)$.

Proof. In view of Remark 2.5, we can recycle the proof of Theorem 4.1. Note that in that proof we nowhere used the fact that $\boldsymbol{g}$ is a rate vector, but only that $\boldsymbol{g}(x t)=$ $\boldsymbol{x}^{\boldsymbol{B}} \boldsymbol{g}(t)+o\left(g_{n}(t)\right)$ for all $x \in(0, \infty)$ and some square matrix $\boldsymbol{B}$ as well as regular variation of $\left|g_{n}\right|$.

THEOREM 4.3 (Representation theorem for $R V_{\boldsymbol{B}}$ ). Let $\boldsymbol{g}$ be a rate vector of length $n$ and let $\boldsymbol{B} \in \mathbb{R}^{n \times n}$ be an index matrix. Then $\boldsymbol{g} \in R V_{\boldsymbol{B}}$ if and only there exist $a \in(0, \infty)$, $\boldsymbol{v} \in \mathbb{R}^{n \times 1}$ and measurable functions $\boldsymbol{\eta}, \boldsymbol{\phi}:[a, \infty) \rightarrow \mathbb{R}^{n \times 1}$, both o $\left(g_{n}(t)\right)$, such that

$$
\boldsymbol{g}(t)=t^{\boldsymbol{B}} \boldsymbol{v}+\boldsymbol{\eta}(t)+t^{\boldsymbol{B}} \int_{a}^{t} u^{-\boldsymbol{B}} \boldsymbol{\phi}(u) u^{-1} \mathrm{~d} u, \quad t \in[a, \infty) .
$$

Proof. Necessity. Assume that $\boldsymbol{g} \in R V_{\boldsymbol{B}}$. Since the functions $\left|g_{i}\right|$ are regularly varying, they are locally bounded on $[a, \infty)$ for some sufficiently large $a \in(0, \infty)$. Define

$$
\begin{aligned}
\boldsymbol{v} & =\int_{a}^{e a} u^{-\boldsymbol{B}} \boldsymbol{g}(u) u^{-1} \mathrm{~d} u, \\
\boldsymbol{\eta}(t) & =\int_{1}^{e}\left\{\boldsymbol{g}(t)-u^{-B} \boldsymbol{g}(u t)\right\} u^{-1} \mathrm{~d} u, \\
\boldsymbol{\phi}(t) & =e^{-B} \boldsymbol{g}(e t)-\boldsymbol{g}(t) .
\end{aligned}
$$

By Theorem 4.1, the vector valued functions $\boldsymbol{\eta}(t)$ and $\boldsymbol{\phi}(t)$ are both $o\left(g_{n}(t)\right)$. The equality (4.1) can be verified by simple algebra.

Sufficiency. Suppose that the rate vector $\boldsymbol{g}$ admits the representation 4.1) with $\boldsymbol{\eta}(t)$ and $\phi(t)$ both $o\left(g_{n}(t)\right)$. Put $b=B_{n n}$. Since $\boldsymbol{B}$ is upper triangular, row $n$ of (4.1) reads

$$
g_{n}(t)=t^{b} v_{n}+\eta_{n}(t)+t^{b} \int_{a}^{t} u^{-b} \phi_{n}(u) u^{-1} \mathrm{~d} u, \quad t \in[a, \infty) .
$$


For $u \in(1, \infty)$ and $t \in[a, \infty)$, we have

$$
\begin{aligned}
g_{n}(u t)-u^{b} g_{n}(t) & =\eta_{n}(u t)-u^{b} \eta_{n}(t)+(u t)^{b} \int_{t}^{u t} y^{-b-1} \phi_{n}(y) \mathrm{d} y \\
& =\eta_{n}(u t)-u^{b} \eta_{n}(t)+u^{b} \int_{1}^{u} y^{-b-1} \phi_{n}(y t) \mathrm{d} y .
\end{aligned}
$$

Fix $x \in(1, \infty)$. We have

$$
\begin{aligned}
\sup _{u \in[1, x]}\left|\eta_{n}(u t)\right| & =\sup _{u \in[1, x]} \frac{\left|\eta_{n}(u t)\right|}{\left|g_{n}(u t)\right|}\left|g_{n}(u t)\right| \\
& \leqslant \sup _{u \in[1, x]} \frac{\left|\eta_{n}(u t)\right|}{\left|g_{n}(u t)\right|} \cdot \sup _{u \in[1, x]}\left|g_{n}(u t)\right|,
\end{aligned}
$$

and thus

$$
\sup _{u \in[1, x]}\left|\eta_{n}(u t)\right|=o\left(\sup _{u \in[1, x]}\left|g_{n}(u t)\right|\right) .
$$

Similarly for $\eta_{n}$ replaced by $\phi_{n}$. In view of 4.2 , it follows that

$$
\sup _{u \in[1, x]}\left|g_{n}(t u)\right| \leqslant x^{b}\left|g_{n}(t)\right|+o\left(\sup _{u \in[1, x]}\left|g_{n}(u t)\right|\right),
$$

and thus

$$
\sup _{u \in[1, x]}\left|g_{n}(t u)\right|=O\left(g_{n}(t)\right) .
$$

Now let us look at the complete rate vector $\boldsymbol{g}$. As in $(4.2)$, we find, again for $x \in(1, \infty)$,

$$
\boldsymbol{g}(x t)-x^{\boldsymbol{B}} \boldsymbol{g}(t)=\boldsymbol{\eta}(x t)-x^{\boldsymbol{B}} \boldsymbol{\eta}(t)+x^{\boldsymbol{B}} \int_{1}^{x} u^{-\boldsymbol{B}} \boldsymbol{\phi}(u t) u^{-1} \mathrm{~d} u .
$$

From 4.3 and the assumption that both $\boldsymbol{\eta}(t)$ and $\boldsymbol{\phi}(t)$ are $o\left(g_{n}(t)\right)$, the above display implies

$$
\boldsymbol{g}(x t)-x^{\boldsymbol{B}} \boldsymbol{g}(t)=o\left(g_{n}(t)\right), \quad x \in[1, \infty) .
$$

Since $x^{\boldsymbol{B}}$ is invertible, the above display and Proposition 2.2 imply that $\boldsymbol{g} \in R V_{\boldsymbol{B}}$.

REMARK 4.4. The case $n=1$ in Theorem 4.3 seems to be a new representation for regularly varying functions. The representation is the same as the one for the class $o \Pi_{g}$ in Bingham et al. (1987, Theorem 3.6.1), but with the difference that the function $g$ is not assumed to be of bounded increase. Indeed, the main point in the proof of sufficiency was precisely to show that the representation actually implies that $g$ is of bounded increase, see 4.3.

THEOREM 4.5 (Representation theorem for $G R V(\boldsymbol{g})$ ). Let $\boldsymbol{g} \in R V_{\boldsymbol{B}}$ and let $f$ be a measurable, real-valued function defined in a neighbourhood of infinity. Then $f \in G R V(\boldsymbol{g})$ with $\boldsymbol{g}$-index $\boldsymbol{c} \in \mathbb{R}^{1 \times n}$ if and only if there exist constants $a \in(0, \infty)$ and $v \in \mathbb{R}$ as well as measurable functions $\eta, \phi:[a, \infty) \rightarrow \mathbb{R}$, both o $\left(g_{n}(t)\right)$, such that

$$
f(t)=v+\eta(t)+\int_{a}^{t}\{\boldsymbol{c g}(u)+\phi(u)\} u^{-1} \mathrm{~d} u, \quad t \in[a, \infty) .
$$


Proof. Let $a \in(0, \infty)$ be large enough so that the domain of $\boldsymbol{g}$ includes $[a, \infty)$ and put

$$
\begin{aligned}
\tilde{f}(t) & =\boldsymbol{c} \int_{a}^{t} \boldsymbol{g}(u) u^{-1} \mathrm{~d} u, \\
\xi(t) & =f(t)-\tilde{f}(t),
\end{aligned}
$$

for $t \in[a, \infty)$. For $x \in(0, \infty)$ and $t$ large enough so that $t \geqslant a$ and $x t \geqslant a$,

$$
\tilde{f}(x t)-\tilde{f}(t)=\boldsymbol{c} \int_{t}^{x t} \boldsymbol{g}(u) u^{-1} \mathrm{~d} u=\boldsymbol{c} \int_{1}^{x} \boldsymbol{g}(u t) u^{-1} \mathrm{~d} u .
$$

By the Uniform convergence theorem for $R V_{\boldsymbol{B}}$,

$$
\tilde{f}(x t)-\tilde{f}(t)=\boldsymbol{c} \int_{1}^{x} u^{B} u^{-1} \mathrm{~d} u \boldsymbol{g}(t)+o\left(g_{n}(t)\right), \quad x \in(0, \infty),
$$

and thus $\tilde{f} \in G R V(\boldsymbol{g})$ with $\boldsymbol{g}$-index $\boldsymbol{c}$. It follows that $f \in G R V(\boldsymbol{g})$ with $\boldsymbol{g}$-index $\boldsymbol{c}$ if and only if

$$
\xi(x t)-\xi(t)=o\left(g_{n}(t)\right), \quad x \in(0, \infty),
$$

that is, $\xi \in o \prod_{\left|g_{n}\right|}$. The Representation theorem for $o \prod_{\left|g_{n}\right|}$ (Bingham et al., 1987, Theorem 3.6.1) says that the above display is equivalent to the existence of a constant $v \in \mathbb{R}$ and measurable functions $\eta, \phi:[a, \infty) \rightarrow \mathbb{R}$, both $o\left(g_{n}(t)\right)$, such that

$$
\xi(t)=v+\eta(t)+\int_{a}^{t} \phi(u) u^{-1} \mathrm{~d} u .
$$

Since $f=\tilde{f}+\xi$, we arrive at the desired representation.

5. Potter bounds. The representation theorems for $R V_{\boldsymbol{B}}$ and $G R V(\boldsymbol{g})$ allow us to derive global upper bounds for $\left\|\boldsymbol{g}(x t)-x^{\boldsymbol{B}} \boldsymbol{g}(t)\right\| /\left|g_{n}(t)\right|$ and $|f(x t)-f(t)-\boldsymbol{h}(x) \boldsymbol{g}(t)| /\left|g_{n}(t)\right|$. In analogy to classical regular variation theory, such bounds will be called Potter bounds.

First recall that for any matrix $Q \in \mathbb{R}^{n \times n}$ and any matrix norm $\|\cdot\|$,

$$
\lim _{m \rightarrow \infty}\left\|\boldsymbol{Q}^{m}\right\|^{1 / m}=\max \{|\lambda|: \lambda \text { is an eigenvalue of } \boldsymbol{Q}\} .
$$

Now let $\boldsymbol{B} \in \mathbb{R}^{n \times n}$ be an upper triangular matrix whose diagonal elements $b_{i}=B_{i i}$, $i \in\{1, \ldots, n\}$, are non-increasing, $b_{1} \geqslant \cdots \geqslant b_{n}$. For $x \in(0, \infty)$, the eigenvalues of $x^{B}$ and $x^{-\boldsymbol{B}}$ are $\left\{x^{b_{i}}\right\}$ and $\left\{x^{-b_{i}}\right\}$, respectively. The above display then implies

$$
\begin{aligned}
& \lim _{x \rightarrow \infty} \frac{\log \left\|x^{\boldsymbol{B}}\right\|}{\log x}=b_{1}, \\
& \lim _{x \rightarrow 0} \frac{\log \left\|x^{\boldsymbol{B}}\right\|}{\log x}=\lim _{y \rightarrow \infty} \frac{\log \left\|y^{-\boldsymbol{B}}\right\|}{-\log y}=b_{n} .
\end{aligned}
$$

TheOREm 5.1 (Potter bounds for $R V_{\boldsymbol{B}}$ ). Let $\boldsymbol{g} \in R V_{\boldsymbol{B}}$. For every $\varepsilon>0$, there exists $t(\varepsilon)>0$ such that

$$
\frac{\left\|\boldsymbol{g}(x t)-x^{\boldsymbol{B}} \boldsymbol{g}(t)\right\|}{\left|g_{n}(t)\right|} \leqslant \begin{cases}\varepsilon x^{b_{1}+\varepsilon} & \text { if } t \geqslant t(\varepsilon) \text { and } x \geqslant 1 \\ \varepsilon x^{b_{n}-\varepsilon} & \text { if } t \geqslant t(\varepsilon) \text { and } t(\varepsilon) / t \leqslant x \leqslant 1 .\end{cases}
$$


Proof. We shall prove the following two statements, which are equivalent to (5.3): for $\delta>0$

$$
\begin{gathered}
\lim _{t \rightarrow \infty} \sup _{x \geqslant 1} \frac{\left\|\boldsymbol{g}(x t)-x^{\boldsymbol{B}} \boldsymbol{g}(t)\right\|}{x^{b_{1}+\delta}\left|g_{n}(t)\right|}=0, \\
\lim _{t \rightarrow \infty} \sup _{x \geqslant 1} \frac{\left\|\boldsymbol{g}(t)-x^{-\boldsymbol{B}} \boldsymbol{g}(x t)\right\|}{x^{-b_{n}+\delta}\left|g_{n}(x t)\right|}=0 .
\end{gathered}
$$

Let $a, \boldsymbol{\eta}$ and $\boldsymbol{\phi}$ be as in the Representation theorem for $R V_{\boldsymbol{B}}$ (Theorem 4.3).

Proof of 5.4. For all $t \geqslant a$ and $x \geqslant 1$,

$$
\begin{aligned}
\boldsymbol{g}(x t)-x^{\boldsymbol{B}} \boldsymbol{g}(t) & =\boldsymbol{\eta}(x t)-x^{\boldsymbol{B}} \boldsymbol{\eta}(t)+(x t)^{\boldsymbol{B}} \int_{t}^{x t} u^{-\boldsymbol{B}} \boldsymbol{\phi}(u) u^{-1} \mathrm{~d} u \\
& =\boldsymbol{\eta}(x t)-x^{\boldsymbol{B}} \boldsymbol{\eta}(t)+x^{\boldsymbol{B}} \int_{1}^{x} u^{-\boldsymbol{B}} \boldsymbol{\phi}(u t) u^{-1} \mathrm{~d} u,
\end{aligned}
$$

and thus, since $b_{n} \leqslant b_{1}$,

$$
\begin{aligned}
\frac{\left\|\boldsymbol{g}(x t)-x^{\boldsymbol{B}} \boldsymbol{g}(t)\right\|}{x^{b_{1}+\delta}\left|g_{n}(t)\right|} \leqslant \frac{\|\boldsymbol{\eta}(x t)\|}{\left|g_{n}(x t)\right|} & \frac{\left|g_{n}(x t)\right|}{x^{b_{n}+\delta}\left|g_{n}(t)\right|}+\frac{\left\|x^{\boldsymbol{B}}\right\|}{x^{b_{1}+\delta}} \frac{\|\boldsymbol{\eta}(t)\|}{\left|g_{n}(t)\right|} \\
& +\frac{\left\|x^{\boldsymbol{B}}\right\|}{x^{b_{1}+\delta / 2}} \int_{1}^{x} \frac{\left\|u^{-\boldsymbol{B}}\right\|}{u^{-b_{n}} x^{\delta / 4}} \frac{\|\boldsymbol{\phi}(u t)\|}{\left|g_{n}(u t)\right|} \frac{\left|g_{n}(u t)\right|}{u^{b_{n}} x^{\delta / 4}\left|g_{n}(t)\right|} u^{-1} \mathrm{~d} u .
\end{aligned}
$$

As a consequence, for $t \geqslant a$,

$$
\begin{aligned}
\sup _{x \geqslant 1} & \frac{\left\|\boldsymbol{g}(x t)-x^{\boldsymbol{B}} \boldsymbol{g}(t)\right\|}{x^{b_{1}+\delta}\left|g_{n}(t)\right|} \\
\leqslant & \sup _{s \geqslant t} \frac{\|\boldsymbol{\eta}(s)\|}{\left|g_{n}(s)\right|} \cdot \sup _{x \geqslant 1} \frac{\left|g_{n}(x t)\right|}{x^{b_{n}+\delta}\left|g_{n}(t)\right|}+\sup _{x \geqslant 1} \frac{\left\|x^{\boldsymbol{B}}\right\|}{x^{b_{1}+\delta}} \cdot \frac{\|\boldsymbol{\eta}(t)\|}{\left|b_{n}(t)\right|} \\
& \quad+\sup _{x \geqslant 1} \frac{\left\|x^{\boldsymbol{B}}\right\|}{x^{b_{1}+\delta / 4}} \cdot \sup _{u \geqslant 1} \frac{\left\|u^{-\boldsymbol{B}}\right\|}{u^{-b_{n}+\delta / 4}} \cdot \sup _{s \geqslant t} \frac{\|\boldsymbol{\phi}(s)\|}{\left|g_{n}(s)\right|} \cdot \sup _{u \geqslant 1} \frac{\left|g_{n}(u t)\right|}{u^{b_{n}+\delta / 4}\left|g_{n}(t)\right|} \cdot \sup _{x \geqslant 1} \frac{\log x}{x^{\delta / 4}} .
\end{aligned}
$$

By Potter's theorem for $R V_{b_{n}}$ and by $5.1-(5.2)$, for every $\varepsilon>0$,

$$
\lim _{t \rightarrow \infty} \sup _{x \geqslant 1} \frac{\left|g_{n}(x t)\right|}{x^{b_{n}+\varepsilon}\left|g_{n}(t)\right|}=1, \quad \sup _{x \geqslant 1} \frac{\left\|x^{\boldsymbol{B}}\right\|}{x^{b_{1}+\varepsilon}}<\infty, \quad \sup _{x \geqslant 1} \frac{\left\|x^{-\boldsymbol{B}}\right\|}{x^{-b_{n}+\varepsilon}}<\infty .
$$

Combine the last two displays and the fact that both $\|\boldsymbol{\eta}(t)\|$ and $\|\boldsymbol{\phi}(t)\|$ are $o\left(g_{n}(t)\right)$ to arrive at (5.4).

Proof of (5.5). By 4.1), for all $t \geqslant a$ and $x \geqslant 1$,

$$
\begin{aligned}
\boldsymbol{g}(t)-x^{-\boldsymbol{B}} \boldsymbol{g}(x t) & =\boldsymbol{\eta}(t)-x^{-\boldsymbol{B}} \boldsymbol{\eta}(x t)+t^{\boldsymbol{B}} \int_{t}^{x t} u^{-\boldsymbol{B}} \boldsymbol{\phi}(u) u^{-1} \mathrm{~d} u \\
& =\boldsymbol{\eta}(t)-x^{-\boldsymbol{B}} \boldsymbol{\eta}(x t)+\int_{1}^{x} u^{-\boldsymbol{B}} \boldsymbol{\phi}(u t) u^{-1} \mathrm{~d} u
\end{aligned}
$$


and thus

$$
\begin{aligned}
\frac{\left\|\boldsymbol{g}(t)-x^{-\boldsymbol{B}} \boldsymbol{g}(x t)\right\|}{x^{-b_{n}+\delta}\left|g_{n}(x t)\right|} & \leqslant \frac{\|\boldsymbol{\eta}(t)\|}{\left|g_{n}(t)\right|} \frac{\left|g_{n}(t)\right|}{x^{-b_{n}+\delta}\left|g_{n}(x t)\right|}+\frac{\left\|x^{-\boldsymbol{B}}\right\|}{x^{-b_{n}+\delta}} \frac{\|\boldsymbol{\eta}(x t)\|}{\left|g_{n}(x t)\right|} \\
& +\frac{\left|g_{n}(t)\right|}{x^{-b_{n}+\delta / 2}\left|g_{n}(x t)\right|} \int_{1}^{x} \frac{\left\|u^{-\boldsymbol{B}}\right\|}{u^{-b_{n}} x^{\delta / 4}} \frac{\|\boldsymbol{\phi}(u t)\|}{\left|g_{n}(u t)\right|} \frac{\left|g_{n}(u t)\right|}{u^{b_{n}} x^{\delta / 4}\left|g_{n}(t)\right|} u^{-1} \mathrm{~d} u .
\end{aligned}
$$

As a consequence, for $t \geqslant a$,

$$
\begin{aligned}
\sup _{x \geqslant 1} & \frac{\left\|\boldsymbol{g}(t)-x^{-\boldsymbol{B}} \boldsymbol{g}(x t)\right\|}{x^{-b_{n}+\delta}\left|g_{n}(x t)\right|} \\
& \leqslant \frac{\|\boldsymbol{\eta}(t)\|}{\left|g_{n}(t)\right|} \cdot \sup _{x \geqslant 1} \frac{\left|g_{n}(t)\right|}{x^{-b_{n}+\delta}\left|g_{n}(x t)\right|}+\sup _{x \geqslant 1} \frac{\left\|x^{-\boldsymbol{B}}\right\|}{x^{-b_{n}+\delta}} \cdot \sup _{s \geqslant t} \frac{\|\boldsymbol{\eta}(s)\|}{\left|g_{n}(s)\right|} \\
+ & \sup _{x \geqslant 1} \frac{\left|g_{n}(t)\right|}{x^{-b_{n}+\delta / 4}\left|g_{n}(x t)\right|} \cdot \sup _{u \geqslant 1} \frac{\left\|u^{-\boldsymbol{B}}\right\|}{u^{-b_{n}+\delta / 4}} \cdot \sup _{s \geqslant t} \frac{\|\boldsymbol{\phi}(s)\|}{\left|g_{n}(s)\right|} \cdot \sup _{u \geqslant 1} \frac{\left|g_{n}(u t)\right|}{u^{b_{n}+\delta / 4}\left|g_{n}(t)\right|} \cdot \sup _{x \geqslant 1} \frac{\log x}{x^{\delta / 4}} .
\end{aligned}
$$

By Potter's theorem for $R V_{b_{n}}$ and by $(5.2)$, for every $\varepsilon>0$,

$$
\lim _{t \rightarrow \infty} \sup _{x \geqslant 1} \frac{\left|g_{n}(t)\right|}{x^{-b_{n}+\varepsilon}\left|g_{n}(x t)\right|}=1, \quad \lim _{t \rightarrow \infty} \sup _{x \geqslant 1} \frac{\left|g_{n}(x t)\right|}{x^{b_{n}+\varepsilon}\left|g_{n}(t)\right|}=1, \quad \sup _{u \geqslant 1} \frac{\left\|u^{-\boldsymbol{B}}\right\|}{u^{-b_{n}+\varepsilon}}<\infty .
$$

Combine the last two displays and the fact that both $\|\boldsymbol{\eta}(t)\|$ and $\|\boldsymbol{\phi}(t)\|$ are $o\left(g_{n}(t)\right)$ to arrive at 5.5.

REMARK 5.2. (a) Since

$$
\frac{\left\|\boldsymbol{g}(t)-x^{-\boldsymbol{B}} \boldsymbol{g}(x t)\right\|}{x^{\delta}\left|g_{n}(t)\right|}=\frac{\left|g_{n}(x t)\right|}{x^{b_{n}+\delta / 2}\left|g_{n}(t)\right|} \cdot \frac{\left\|\boldsymbol{g}(t)-x^{-\boldsymbol{B}} \boldsymbol{g}(x t)\right\|}{x^{-b_{n}+\delta / 2}\left|g_{n}(x t)\right|},
$$

equation 5.5 and Potter's theorem for $R V_{b_{n}}$ imply

$$
\lim _{t \rightarrow \infty} \sup _{x \geqslant 1} \frac{\left\|\boldsymbol{g}(t)-x^{-\boldsymbol{B}} \boldsymbol{g}(x t)\right\|}{x^{\delta}\left|g_{n}(t)\right|}=0, \quad \delta>0 .
$$

(b) Equation (5.4) can be used to give a simple proof of a statement which is slightly weaker than (5.5). For $t \geqslant a$ and $x \geqslant 1$,

$$
\frac{\left\|\boldsymbol{g}(t)-x^{-\boldsymbol{B}} \boldsymbol{g}(x t)\right\|}{x^{b_{1}-2 b_{n}+\delta}\left|g_{n}(x t)\right|} \leqslant \frac{\left\|x^{-\boldsymbol{B}}\right\|}{x^{-b_{n}+\delta / 3}} \cdot \frac{\left|g_{n}(t)\right|}{x^{-b_{n}+\delta / 3}\left|g_{n}(x t)\right|} \cdot \frac{\left\|\boldsymbol{g}(x t)-x^{\boldsymbol{B}} \boldsymbol{g}(t)\right\|}{x^{b_{1}+\delta / 3}\left|g_{n}(t)\right|} .
$$

By $(5.2)$ and by Potter's theorem for $R V_{b_{n}}$, for every $\varepsilon>0$,

$$
\sup _{x \geqslant 1} \frac{\left\|x^{-\boldsymbol{B}}\right\|}{x^{-b_{n}+\varepsilon}}<\infty, \quad \lim _{t \rightarrow \infty} \sup _{x \geqslant 1} \frac{\left|g_{n}(t)\right|}{x^{-b_{n}+\varepsilon}\left|g_{n}(x t)\right|}=1 .
$$

Combine the last two displays and (5.4) to arrive at

$$
\lim _{t \rightarrow \infty} \sup _{x \geqslant 1} \frac{\left\|\boldsymbol{g}(t)-x^{-\boldsymbol{B}} \boldsymbol{g}(x t)\right\|}{x^{b_{1}-2 b_{n}+\delta}\left|g_{n}(x t)\right|}=0, \quad \delta>0 .
$$

For $a, b \in \mathbb{R}$, write $a \vee b=\max (a, b)$. 
THEOREM 5.3 (Potter bounds for $G R V(\boldsymbol{g})$ ). Let $\boldsymbol{g} \in R V_{\boldsymbol{B}}$ and let $f \in G R V(\boldsymbol{g})$. For every $\varepsilon>0$, there exists $t(\varepsilon)>0$ such that

$$
\frac{|f(x t)-f(t)-\boldsymbol{h}(x) \boldsymbol{g}(t)|}{\left|g_{n}(t)\right|} \leqslant \begin{cases}\varepsilon x^{\left(b_{1}+\varepsilon\right) \vee 0} & \text { if } t \geqslant t(\varepsilon) \text { and } x \geqslant 1 \\ \varepsilon x^{\left(b_{n}-\varepsilon\right) \wedge 0} & \text { if } t \geqslant t(\varepsilon) \text { and } t(\varepsilon) / t \leqslant x \leqslant 1 .\end{cases}
$$

Proof. We shall prove the following two statements, which are equivalent to (5.7): for $\delta>0$,

$$
\begin{array}{r}
\lim _{t \rightarrow \infty} \sup _{x \geqslant 1} \frac{|f(x t)-f(t)-\boldsymbol{h}(x) \boldsymbol{g}(t)|}{x^{\left(b_{1}+\delta\right) \vee 0}\left|g_{n}(t)\right|}=0, \\
\lim _{t \rightarrow \infty} \sup _{x \geqslant 1} \frac{\left|f(t)-f(x t)-\boldsymbol{h}\left(x^{-1}\right) \boldsymbol{g}(x t)\right|}{x^{\left(-b_{n}+\delta\right) \vee 0}\left|g_{n}(x t)\right|}=0 .
\end{array}
$$

Recall that $\boldsymbol{h}(x)=\boldsymbol{c} \int_{1}^{x} u^{\boldsymbol{B}} u^{-1} \mathrm{~d} u$ for $x \in(0, \infty)$, with $\boldsymbol{c} \in \mathbb{R}^{1 \times n}$ the $\boldsymbol{g}$-index of $f$. Also, recall the representation of $f$ in Theorem 4.5 .

Proof of 5.8. For $t \geqslant a$ and $x \geqslant 1$,

$$
\begin{aligned}
f(x t)-f(t)- & \boldsymbol{h}(x) \boldsymbol{g}(t) \\
& =\boldsymbol{c} \int_{1}^{x}\left\{\boldsymbol{g}(u t)-u^{B} \boldsymbol{g}(t)\right\} u^{-1} \mathrm{~d} u+\eta(x t)-\eta(t)+\int_{1}^{x} \phi(u t) u^{-1} \mathrm{~d} u
\end{aligned}
$$

and thus, since $b_{n}+\delta \leqslant b_{1}+\delta \leqslant\left(b_{1}+\delta\right) \vee 0$,

$$
\begin{aligned}
& \frac{|f(x t)-f(t)-\boldsymbol{h}(x) \boldsymbol{g}(t)|}{x^{\left(b_{1}+\delta\right) \vee 0}\left|g_{n}(t)\right|} \\
& \leqslant \frac{\|\boldsymbol{c}\|}{x^{\left(b_{1}+\delta\right) \vee 0}} \int_{1}^{x} \frac{\left\|\boldsymbol{g}(u t)-u^{B} \boldsymbol{g}(t)\right\|}{u^{b_{1}+\delta / 2}\left|g_{n}(t)\right|} u^{b_{1}+\delta / 2-1} \mathrm{~d} u+\frac{|\eta(x t)|}{\left|g_{n}(x t)\right|} \cdot \frac{\left|g_{n}(x t)\right|}{x^{b_{n}+\delta}\left|g_{n}(t)\right|}+\frac{|\eta(t)|}{\left|g_{n}(t)\right|} \\
& \quad+\frac{1}{x^{\left(b_{1}+\delta\right) \vee 0}} \int_{1}^{x} \frac{|\phi(u t)|}{\left|g_{n}(u t)\right|} \frac{\left|g_{n}(u t)\right|}{u^{b_{n}+\delta / 2}\left|g_{n}(t)\right|} u^{b_{n}+\delta / 2-1} \mathrm{~d} u .
\end{aligned}
$$

We obtain that, for $t \geqslant a$,

$$
\begin{aligned}
& \sup _{x \geqslant 1} \frac{|f(x t)-f(t)-\boldsymbol{h}(x) \boldsymbol{g}(t)|}{x^{\left(b_{1}+\delta\right) \vee 0}\left|g_{n}(t)\right|} \\
& \leqslant \sup _{u \geqslant 1} \frac{\left\|\boldsymbol{g}(u t)-u^{\boldsymbol{B}} \boldsymbol{g}(t)\right\|}{u^{b_{1}+\delta / 2}\left|g_{n}(t)\right|} \cdot \sup _{x \geqslant 1} \frac{\|\boldsymbol{c}\|}{x^{\left(b_{1}+\delta\right) \vee 0}} \int_{1}^{x} u^{b_{1}+\delta / 2-1} \mathrm{~d} u \\
& \quad+\sup _{s \geqslant 1} \frac{|\eta(s)|}{\left|g_{n}(s)\right|} \cdot \sup _{x \geqslant 1} \frac{\left|g_{n}(x t)\right|}{x^{b_{n}+\delta}\left|g_{n}(t)\right|}+\frac{|\eta(t)|}{\left|g_{n}(t)\right|} \\
& \quad+\sup _{s} \frac{|\phi(s)|}{\left|g_{n}(s)\right|} \cdot \sup _{u \geqslant 1} \frac{\left|g_{n}(u t)\right|}{u^{b_{n}+\delta / 2}\left|g_{n}(t)\right|} \cdot \sup _{x \geqslant 1} \frac{1}{x^{\left(b_{1}+\delta\right) \vee 0}} \int_{1}^{x} u^{b_{n}+\delta / 2-1} \mathrm{~d} u .
\end{aligned}
$$

In view of Potter's theorem for $R V_{b_{n}}$ and for $R V_{\boldsymbol{B}}$ (Theorem 5.1), it suffices to note that $\int_{1}^{x} u^{b_{1}+\delta / 2-1} \mathrm{~d} u=O\left(x^{\left(b_{1}+\delta\right) \vee 0}\right)$ as $x \rightarrow \infty$. 
Proof of (5.9). By 2.6), $\boldsymbol{h}\left(x^{-1}\right)=-\boldsymbol{h}(x) x^{-\boldsymbol{B}}$ and thus

$$
\begin{aligned}
\boldsymbol{h}\left(x^{-1}\right) \boldsymbol{g}(x t)=-\boldsymbol{h}(x) x^{-\boldsymbol{B}} \boldsymbol{g}(x t) & \\
& =-\boldsymbol{c} \int_{1}^{x} u^{\boldsymbol{B}} u^{-1} \mathrm{~d} u x^{-\boldsymbol{B}} \boldsymbol{g}(x t)=-\boldsymbol{c} \int_{1}^{x}(x / u)^{-\boldsymbol{B}} \boldsymbol{g}(x t) u^{-1} \mathrm{~d} u
\end{aligned}
$$

for $x \geqslant 1$ and $t \geqslant a$. We obtain that for such $x$ and $t$,

$$
\begin{aligned}
f(t)-f(x t) & -\boldsymbol{h}\left(x^{-1}\right) \boldsymbol{g}(x t) \\
& =\eta(t)-\eta(x t)-\int_{1}^{x} \phi(u t) u^{-1} \mathrm{~d} u-\boldsymbol{c} \int_{1}^{x}\left\{\boldsymbol{g}(u t)-(x / u)^{-\boldsymbol{B}} \boldsymbol{g}(x t)\right\} u^{-1} \mathrm{~d} u
\end{aligned}
$$

whence

$$
\begin{aligned}
& \frac{\left|f(t)-f(x t)-\boldsymbol{h}\left(x^{-1}\right) \boldsymbol{g}(x t)\right|}{x^{\left(-b_{n}+\delta\right) \vee 0}\left|g_{n}(x t)\right|} \\
& \leqslant \frac{|\eta(t)|}{\left|g_{n}(t)\right|} \cdot \frac{\left|g_{n}(t)\right|}{x^{-b_{n}+\delta}\left|g_{n}(x t)\right|}+\frac{|\eta(x t)|}{\left|g_{n}(x t)\right|} \\
& \quad+\frac{1}{x^{\left\{\left(-b_{n}+\delta\right) \vee 0\right\}+b_{n}-\delta / 2}} \cdot \int_{1}^{x} \frac{|\phi(u t)|}{\left|g_{n}(u t)\right|} \frac{\left|g_{n}(u t)\right|}{(x / u)^{-b_{n}+\delta / 2}\left|g_{n}(x t)\right|} u^{b_{n}-\delta / 2-1} \mathrm{~d} u \\
& +\frac{\|\boldsymbol{c}\|}{x^{\left\{\left(-b_{n}+\delta\right) \vee 0\right\}+b_{n}-\delta / 2}} \\
& \quad \cdot \int_{1}^{x} \frac{\left\|\boldsymbol{g}(u t)-(x / u)^{-\boldsymbol{B}} \boldsymbol{g}(x t)\right\|}{(x / u)^{\delta / 4}\left|g_{n}(u t)\right|} \frac{\left|g_{n}(u t)\right|}{(x / u)^{-b_{n}+\delta / 4}\left|g_{n}(x t)\right|} u^{b_{n}-\delta / 2-1} \mathrm{~d} u .
\end{aligned}
$$

Since $\left\{\left(-b_{n}+\delta\right) \vee 0\right\}+b_{n}-\delta / 2=\left(b_{n}-\delta / 2\right) \vee(\delta / 2)$, we find, for $t \geqslant a$,

$$
\begin{aligned}
& \sup _{x \geqslant 1} \frac{\left|f(t)-f(x t)-\boldsymbol{h}\left(x^{-1}\right) \boldsymbol{g}(x t)\right|}{x^{\left(-b_{n}+\delta\right) \vee 0}\left|g_{n}(x t)\right|} \\
& \leqslant \frac{|\eta(t)|}{\left|g_{n}(t)\right|} \cdot \sup _{x \geqslant 1} \frac{\left|g_{n}(t)\right|}{x^{-b_{n}+\delta}\left|g_{n}(x t)\right|}+\sup _{s \geqslant 1} \frac{|\eta(s)|}{\left|g_{n}(s)\right|} \\
& \quad+\sup _{s \geqslant 1} \frac{|\phi(s)|}{\left|g_{n}(s)\right|} \cdot \sup _{s \geqslant t} \sup _{y \geqslant 1} \frac{\left|g_{n}(s)\right|}{y^{-b_{n}+\delta / 2}\left|g_{n}(y s)\right|} \cdot \frac{1}{x^{\left(b_{n}-\delta / 2\right) \vee(\delta / 2)}} \int_{1}^{x} u^{b_{n}-\delta / 2-1} \mathrm{~d} u \\
& \quad+\sup _{s \geqslant 1} \sup _{y \geqslant 1} \frac{\| \boldsymbol{g}(s)-y^{-B} \boldsymbol{g}(y s)||}{y^{\delta / 4}\left|g_{n}(s)\right|} \cdot \operatorname{supsup}_{s \geqslant 1} \frac{\mid g_{n}(s)}{y^{-b_{n}+\delta / 4}\left|g_{n}(y s)\right|} \\
& \quad \cdot \frac{1}{x^{\left(b_{n}-\delta / 2\right) \vee(\delta / 2)}} \int_{1}^{x} u^{b_{n}-\delta / 2-1} \mathrm{~d} u .
\end{aligned}
$$

Now apply the following elements:

(a) the assumption that both $\eta(t)$ and $\phi(t)$ are $o\left(g_{n}(t)\right)$ (Theorem 4.5);

(b) Potter's theorem for $R V_{b_{n}}$;

(c) Potter's theorem for $R V_{\boldsymbol{B}}$ [equation $(5.6)$ in Remark 5.2;

(d) the fact that for $a \in \mathbb{R}$ and $\varepsilon>0, \int_{1}^{x} u^{a-1} \mathrm{~d} u=O\left(x^{a \vee \varepsilon}\right)$ as $x \rightarrow \infty$;

to arrive at 5.9 .

6. Special cases. For $\boldsymbol{g} \in R V_{\boldsymbol{B}}$, the diagonal elements of $\boldsymbol{B}$ are the indices of regular variation of the component functions $\left|g_{i}\right|$. The form and behaviour of $\boldsymbol{g} \in R V_{\boldsymbol{B}}$ and 
$f \in G R V(\boldsymbol{g})$ depend strongly on the values of these indices. In this section, we consider two special cases: first the case where all indices are different from zero, 6.1 , and second the case where all indices are distinct, 6.2 .

The special case where all indices are equal to zero gives rise to functions $f$ that are $\Pi$-regularly varying of order $n$, generalizing the function class $\Pi$ in de Haan (1970) $(n=1)$ and the function class $\Pi$ with remainder in Omey and Willekens (1988) $(n=2)$. The theory for this class is worked out in Omey and Segers (2009, sections 5-6). This case turns out to be rather diverse, including for instance the Lambert $W$ function and the inverse of the reciprocal of the complementary error function (Omey and Segers, 2009, section 8).

6.1. All indices different from zero. Let $\boldsymbol{g} \in R V_{\boldsymbol{B}}$. If none of the diagonal elements of $\boldsymbol{B}$ are zero, that is, if none of the rate functions $g_{i}$ are slowly varying, then the class $G R V(\boldsymbol{g})$ essentially consists of linear combinations of the rate functions and the constant function.

ThEOREM 6.1. Let $\boldsymbol{B} \in \mathbb{R}^{n \times n}$ be upper triangular and invertible, i.e. without zeros on the diagonal. Let $\boldsymbol{g} \in R V_{\boldsymbol{B}}$. Then $f \in G R V(\boldsymbol{g})$ with $\boldsymbol{g}$-index $\boldsymbol{c}$ if and only if there exists a constant $C$ such that

$$
f(t)=C+\boldsymbol{c} \boldsymbol{B}^{-1} \boldsymbol{g}(t)+o\left(g_{n}(t)\right) .
$$

Proof. Necessity. Suppose $f \in G R V(\boldsymbol{g})$ with $\boldsymbol{g}$-index $\boldsymbol{c}$. Define $\xi(t)=f(t)-\boldsymbol{c} \boldsymbol{B}^{-1} \boldsymbol{g}(t)$. Then for $x \in(0, \infty)$, in view of A.5,

$$
\begin{aligned}
\xi(x t) & =f(x t)-\boldsymbol{c} \boldsymbol{B}^{-1} \boldsymbol{g}(x t) \\
& =f(t)+\boldsymbol{c} \boldsymbol{B}^{-1}\left(x^{\boldsymbol{B}}-\boldsymbol{I}\right) \boldsymbol{g}(t)-\boldsymbol{c} \boldsymbol{B}^{-1} x^{\boldsymbol{B}} \boldsymbol{g}(t)+o\left(g_{n}(t)\right) \\
& =f(t)-\boldsymbol{c} \boldsymbol{B}^{-1} \boldsymbol{g}(t)+o\left(g_{n}(t)\right) \\
& =\xi(t)+o\left(g_{n}(t)\right) .
\end{aligned}
$$

Equation (6.1) now follows from the representation theorem for $o \Pi_{g}$ for auxiliary functions which are regularly varying but not slowly varying (Bingham et al., 1987, Theorems 3.6.1 $1^{ \pm}$, pp. $\left.152-153\right)$. Note that in case $b_{n}>0$, the constant $C$ can be absorbed in the $o\left(g_{n}(t)\right)$ remainder term.

Sufficiency. For $f$ as in 6.1 , we have

$$
f(x t)-f(t)=\boldsymbol{c B}^{-1}\left(x^{\boldsymbol{B}}-\boldsymbol{I}\right) \boldsymbol{g}(t)+o\left(g_{n}(t)\right)
$$

so that, by A.5), indeed $f \in G R V(\boldsymbol{g})$ with $\boldsymbol{g}$-index $\boldsymbol{c}$.

EXAmple 6.2 (Power series). Suppose that $f(x)=\sum_{k=0}^{\infty} a_{k} x^{-k \alpha}$ where $\alpha>0$ and $\sum_{k=0}^{\infty} a_{k} z^{k}$ is a power series which is convergent in a neighbourhood of $z=0$. Then for $x>0$ and integer $n \geqslant 1$,

$$
f(x t)-f(t)=\sum_{k=1}^{n} a_{k}\left(x^{-k \alpha}-1\right) t^{-k \alpha}+o\left(t^{-n \alpha}\right) .
$$

The rate vector $\boldsymbol{g}(t)=\left(t^{-\alpha}, t^{-2 \alpha}, \ldots, t^{-n \alpha}\right)^{\prime}$ is regularly varying with index matrix

$$
\boldsymbol{B}=\operatorname{diag}(-\alpha,-2 \alpha, \ldots,-n \alpha) .
$$

By the first display, the function $f$ belongs to $G R V(\boldsymbol{g})$ with $\boldsymbol{g}$-index $\boldsymbol{c}=\left(-k a_{k} \alpha\right)_{k=1}^{n}$. 
6.2. All indices distinct. In case all the diagonal elements of the index matrix $\boldsymbol{B}$ are distinct, rate vectors $\boldsymbol{g}$ in the class $R V_{\boldsymbol{B}}$ are essentially given by linear combinations of power functions and $g_{n}$. This representation can be extended to the class $G R V(\boldsymbol{g})$ with $\boldsymbol{g} \in R V_{\boldsymbol{B}}$. In addition, the Potter bounds for $R V_{\boldsymbol{B}}$ and $G R V(\boldsymbol{g})$ can be sharpened.

For a square matrix $\boldsymbol{A}$, let $\operatorname{diag}(\boldsymbol{A})$ be the diagonal matrix of the same dimension containing the diagonal elements of $\boldsymbol{A}$.

TheOREM 6.3. Let $\boldsymbol{B} \in \mathbb{R}^{n \times n}(n \geqslant 2)$ be upper triangular with distinct diagonal elements $B_{11}>\cdots>B_{n n}$. Put $b_{i}=B_{i i}$ and $\boldsymbol{D}=\operatorname{diag}(\boldsymbol{B}) \in \mathbb{R}^{n \times n}$. Then a rate vector $\boldsymbol{g}$ belongs to $R V_{\boldsymbol{B}}$ if and only if $\left|g_{n}\right| \in R V_{b_{n}}$ and there exists an upper triangular and invertible matrix $\boldsymbol{Q} \in \mathbb{R}^{n \times n}$ such that $\boldsymbol{Q}_{n n}=1, \boldsymbol{B}=\boldsymbol{Q} \boldsymbol{D} \boldsymbol{Q}^{-1}$ and

$$
g_{i}(t)=\sum_{j=i}^{n-1} Q_{i j} t^{b_{j}}+Q_{i n} g_{n}(t)+o\left(g_{n}(t)\right), \quad i \in\{1, \ldots, n-1\} .
$$

Proof. Necessity. Suppose that $\boldsymbol{g} \in R V_{\boldsymbol{B}}$. By Lemma 6.6 below, there exists an invertible, upper triangular matrix $\boldsymbol{P}$ such that $\boldsymbol{B}=\boldsymbol{P} \boldsymbol{D} \boldsymbol{P}^{-1}$ and with $P_{i i}=1$ for all $i$. Then $\tilde{\boldsymbol{g}}:=$ $\boldsymbol{P}^{-1} \boldsymbol{g}$ is a rate vector as well and is regularly varying with index matrix $\boldsymbol{P}^{-1} \boldsymbol{B} \boldsymbol{P}=\boldsymbol{D}$, see Remark 3.5(a). For $x \in(0, \infty)$, the matrix $x^{\boldsymbol{D}}$ is diagonal and with diagonal elements $x^{b_{1}}, \ldots, x^{b_{n}}$. Fix $i \in\{1, \ldots, n-1\}$. Row $i$ of the relation $\tilde{\boldsymbol{g}}(x t)=x^{\boldsymbol{D}} \tilde{\boldsymbol{g}}(t)+o\left(g_{n}(t)\right)$ is just

$$
\tilde{g}_{i}(x t)=x^{b_{i}} \tilde{g}_{i}(t)+o\left(g_{n}(t)\right) .
$$

Writing $L_{i}(t)=t^{-b_{i}} \tilde{g}_{i}(t)$, we find

$$
L_{i}(x t)=L_{i}(t)+o\left(t^{-b_{i}} g_{n}(t)\right) .
$$

Since the function $t^{-b_{i}}\left|g_{n}(t)\right|$ is regularly varying with negative index $b_{n}-b_{i}$, the representation theorem for $o \prod_{g}$ for auxiliary functions $g$ with positive increase (Bingham et al. 1987, Theorem $3.6 .1^{-}$, p. 152) implies that there exists $C_{i} \in \mathbb{R}$ such that

$$
L_{i}(t)=C_{i}+o\left(t^{-b_{i}} g_{n}(t)\right)
$$

and thus

$$
\tilde{g}_{i}(t)=C_{i} t^{b_{i}}+o\left(g_{n}(t)\right) .
$$

Since $\tilde{g}$ is a rate vector, $C_{i}$ must be nonzero. Write $\boldsymbol{C}=\left(C_{1}, \ldots, C_{n-1}, 1\right)$. Since $\boldsymbol{g}=\boldsymbol{P} \tilde{\boldsymbol{g}}$, we find

$$
\boldsymbol{g}(t)=\boldsymbol{P} \boldsymbol{C}\left(t^{b_{1}}, \ldots, t^{b_{n-1}}, g_{n}(t)\right)^{\prime}+o\left(g_{n}(t)\right),
$$

which is 6.2 in matrix notation and with $\boldsymbol{Q}=\boldsymbol{P C}$. Finally, since diagonal matrices

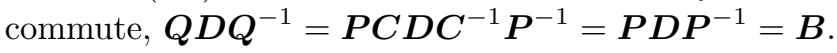

Sufficiency. The rate vector $\hat{\boldsymbol{g}}(t)=\left(t^{b_{1}}, \ldots, t^{b_{n-1}}, g_{n}(t)\right)^{\prime}$ is regularly varying with index matrix $\boldsymbol{D}$. By Remark 3.5 (a), $\boldsymbol{g}=\boldsymbol{Q} \hat{\boldsymbol{g}}$ is regularly varying with index matrix $\boldsymbol{Q} \boldsymbol{D} \boldsymbol{Q}^{-1}=$ $B$.

THEOREM 6.4. Let $\boldsymbol{B} \in \mathbb{R}^{n \times n}(n \geqslant 2)$ be upper triangular with distinct diagonal elements $B_{11}>\cdots>B_{n n}$. Put $b_{i}=B_{i i}$. Then $f \in G R V(\boldsymbol{g})$ for some $\boldsymbol{g} \in R V_{\boldsymbol{B}}$ if and only if 
there exist $a, a_{0}, \ldots, a_{n} \in \mathbb{R}$ such that

$$
f(t)=a_{0}+\sum_{i=1}^{n-1} a_{i} \int_{1}^{t} u^{b_{i}-1} \mathrm{~d} u+\int_{a}^{t}\left\{a_{n}+o(1)\right\} g_{n}(u) u^{-1} \mathrm{~d} u+o\left(g_{n}(t)\right) .
$$

Proof. Let $\hat{\boldsymbol{g}}(t)=\left(t^{b_{1}}, \ldots, t^{b_{n-1}}, g_{n}(t)\right)^{\prime}$. By Theorem 6.3, $\boldsymbol{g}(t)=\boldsymbol{Q} \hat{\boldsymbol{g}}(t)+o\left(g_{n}(t)\right)$ for some invertible, upper triangular matrix $\boldsymbol{Q}$ satisfying $Q_{n n}=1$ and $\boldsymbol{Q} \operatorname{diag}(\boldsymbol{B}) \boldsymbol{Q}^{-1}=\boldsymbol{B}$.

Necessity. Suppose that $f \in G R V(\boldsymbol{g})$ with $\boldsymbol{g}$-index $\boldsymbol{c} \in \mathbb{R}^{1 \times n}$. By the representation theorem for $G R V(\boldsymbol{g})$ (Theorem 4.5), we have

$$
f(t)=v+o\left(g_{n}(t)\right)+\int_{a}^{t}\left\{\boldsymbol{c g}(u)+o\left(g_{n}(u)\right)\right\} u^{-1} \mathrm{~d} u .
$$

Putting $\boldsymbol{a}=\boldsymbol{c} \boldsymbol{Q}$, we find $\boldsymbol{c} \boldsymbol{g}(t)=\boldsymbol{a} \hat{\boldsymbol{g}}(t)+o\left(g_{n}(t)\right)$ and thus

$$
f(t)=v+\sum_{i=1}^{n-1} a_{i} \int_{a}^{t} u^{b_{i}-1} \mathrm{~d} u+\int_{a}^{t}\left\{a_{n}+o(1)\right\} g_{n}(u) u^{-1} \mathrm{~d} u+o\left(g_{n}(t)\right) .
$$

Put $a_{0}=v-\sum_{i=1}^{n-1} a_{i} \int_{1}^{a} u^{b_{i}-1} \mathrm{~d} u$ to arrive at 6.3 .

Sufficiency. Put $\boldsymbol{c}=\left(a_{1}, \ldots, a_{n}\right) \boldsymbol{Q}^{-1}$, do the steps of the previous paragraph in reverse, and apply the representation theorem for $G R V(\boldsymbol{g})$ (Theorem 6.4.

In case $B_{n n} \neq 0$, the representation in 6.3 can be even further simplified to

$$
f(t)=a_{0}+\sum_{i=1}^{n-1} a_{i} \int_{1}^{t} u^{b_{i}-1} \mathrm{~d} u+a_{n} g_{n}(t)+o\left(g_{n}(t)\right)
$$

(where $a_{0}$ and $a_{n}$ have been redefined). Note also that for those $i \in\{1, \ldots, n-1\}$ for which $b_{i}$ is nonzero (which has to occur for all but at most one $i$ ), the integral $\int_{1}^{t} u^{b_{i}-1} \mathrm{~d} u$ can be replaced by $t^{b_{i}}$ (after redefining $a_{i}$ and $a_{0}$ ).

Theorem 6.5 (Improved Potter bounds for $R V_{\boldsymbol{B}}$ and $G R V(\boldsymbol{g})$ ). Let $\boldsymbol{B} \in \mathbb{R}^{n \times n}$ with all diagonal elements distinct. Put $b_{n}=B_{n n}$.

(a) If $\boldsymbol{g} \in R V_{\boldsymbol{B}}$, then for every $\varepsilon>0$, there exists $t(\varepsilon)>0$ such that

$$
\frac{\left\|\boldsymbol{g}(x t)-x^{\boldsymbol{B}} \boldsymbol{g}(t)\right\|}{\left|g_{n}(t)\right|} \leqslant \varepsilon x^{b_{n}} \max \left(x^{\varepsilon}, x^{-\varepsilon}\right), \quad t \geqslant t(\varepsilon), \quad x \geqslant t(\varepsilon) / t .
$$

(b) If $\boldsymbol{g} \in R V_{\boldsymbol{B}}$ and $f \in G R V(\boldsymbol{g})$ with limit functions $\boldsymbol{h}$, then for every $\varepsilon>0$, there exists $t(\varepsilon)>0$ such that

$$
\frac{|f(x t)-f(t)-\boldsymbol{h}(x) \boldsymbol{g}(t)|}{\left|g_{n}(t)\right|} \leqslant \varepsilon \max \left(1, x^{b_{n}+\varepsilon}, x^{b_{n}-\varepsilon}\right) \quad t \geqslant t(\varepsilon), \quad x \geqslant t(\varepsilon) / t .
$$

Proof. (a) Put $b_{i}=B_{i i}$ and $\boldsymbol{D}=\operatorname{diag}(\boldsymbol{B})$. By Theorem 6.3, there exists an invertible, upper triangular matrix $\boldsymbol{Q} \in \mathbb{R}^{n \times n}$ so that $\boldsymbol{B}=\boldsymbol{Q} \boldsymbol{D} \boldsymbol{Q}^{-1}$ and $\boldsymbol{g}=\boldsymbol{Q} \hat{\boldsymbol{g}}$ where $\hat{\boldsymbol{g}}(t)=$ $\left(t^{b_{1}}, \ldots, t^{b_{n-1}}, g_{n}(t)\right)^{\prime}$. Since $x^{\boldsymbol{D}}$ is diagonal with diagonal elements $x^{b_{1}}, \ldots, x^{b_{n}}$, we have

$$
\hat{\boldsymbol{g}}(x t)-x^{\boldsymbol{D}} \hat{\boldsymbol{g}}(t)=\left(0, \ldots, 0, g_{n}(x t)-x^{b_{n}} g_{n}(t)\right)^{\prime}, \quad x \in(0, \infty) .
$$


As a consequence, since $x^{B}=\boldsymbol{Q} x^{D} Q^{-1}$,

$$
\begin{aligned}
\left\|\boldsymbol{g}(x t)-x^{\boldsymbol{B}} \boldsymbol{g}(t)\right\| & =\left\|\boldsymbol{Q} \hat{\boldsymbol{g}}(x t)-\boldsymbol{Q} x^{\boldsymbol{D}} \boldsymbol{Q}^{-1} \boldsymbol{Q} \hat{\boldsymbol{g}}(t)\right\| \\
& \leqslant\|\boldsymbol{Q}\|\left\|\hat{\boldsymbol{g}}(x t)-x^{\boldsymbol{D}} \hat{\boldsymbol{g}}(t)\right\| \\
& =\|Q\|\left|g_{n}(x t)-x^{b_{n}} g_{n}(t)\right| .
\end{aligned}
$$

[here we implicitly assumed, without loss of generality, that the norm of $(0, \ldots, 0,1)$ is equal to unity]. Now apply Potter's theorem for $R V_{b_{n}}$ (Theorem 5.1).

(b) For $x \leqslant 1$, this is just the general Potter bound (5.7). For $x \geqslant 1$, start again from (5.10) and use (6.4) rather than the general Potter bound for $R V_{\boldsymbol{B}}$.

Lemma 6.6. Let $\boldsymbol{B} \in \mathbb{R}^{n \times n}$ be upper triangular and with $n$ distinct diagonal elements. Let $\boldsymbol{D}=\operatorname{diag}(\boldsymbol{B}) \in \mathbb{R}^{n \times n}$. Then for any vector $\boldsymbol{q} \in \mathbb{R}^{n}$ with nonzero elements there exists a unique upper triangular matrix $\boldsymbol{Q} \in \mathbb{R}^{n \times n}$ with diagonal $\boldsymbol{q}$ and such that $\boldsymbol{B}=\boldsymbol{Q D} \boldsymbol{Q}^{-1}$. Proof. The proof is by induction on $n$ and is omitted for brevity.

A. Matrix exponentials. For a square matrix $\boldsymbol{B}$, recall the matrix exponential functions

$$
\exp (\boldsymbol{B})=\sum_{k=0}^{\infty} \frac{1}{k !} \boldsymbol{B}^{k}, \quad \quad x^{\boldsymbol{B}}=\exp \{(\log x) \boldsymbol{B}\},
$$

for $x \in(0, \infty)$. By convention, $\boldsymbol{B}^{0}=\boldsymbol{I}$, the identity matrix. If $\boldsymbol{B}$ is upper triangular, the entries of $x^{B}$ can be computed recursively.

Proposition A.1. Let $\boldsymbol{B} \in \mathbb{R}^{n \times n}$ be upper triangular and put $\boldsymbol{A}(x)=x^{\boldsymbol{B}}, x \in(0, \infty)$. Then $A_{i i}(x)=x^{b_{i}}$ with $b_{i}=B_{i i}, i \in\{1, \ldots, n\}$, while for $i \in\{1, \ldots, n-1\}$,

$$
\left(A_{i, i+1}(x), \ldots, A_{i, n}(x)\right)=x^{b_{i}}\left(B_{i, i+1}, \ldots, B_{i, n}\right) \int_{1}^{x} \boldsymbol{A}_{i+1, n}(y) y^{-b_{i}-1} \mathrm{~d} y,
$$

where $\boldsymbol{A}_{k l}(x)=\left(A_{i j}(x)\right)_{i, j=k}^{l}$. In particular, if all diagonal elements of $\boldsymbol{B}$ are zero, then for integer $i$ and $k$ such that $1 \leqslant i<i+k \leqslant n$ and for $x \in(0, \infty)$,

$$
A_{i, i+k}(x)=\sum_{l=1}^{k} \frac{(\log x)^{l}}{l !} \sum_{i=j_{0}<\cdots<j_{l}=i+k} \prod_{m=1}^{l} B_{j_{m-1}, j_{m}},
$$

the inner sum being over all $(l+1)$-tuples of positive integers $\left(j_{0}, \ldots, j_{l}\right)$ satisfying the stated (in)equalities.

Proof. The starting point is the relation $\boldsymbol{A}(x y)=\boldsymbol{A}(x) \boldsymbol{A}(y)$, from which it follows that $\boldsymbol{A}(x y)-\boldsymbol{A}(x)=\{\boldsymbol{A}(y)-\boldsymbol{I}\} \boldsymbol{A}(x)$. We obtain

$$
\frac{\boldsymbol{A}(x y)-\boldsymbol{A}(x)}{y-1}=\frac{\boldsymbol{A}(y)-I_{n}}{y-1} \boldsymbol{A}(x), \quad x, y \in(0, \infty) .
$$

Taking limits as $y \rightarrow 1$, we find that $x \dot{\boldsymbol{A}}(x)=\boldsymbol{B} \boldsymbol{A}(x)$, and therefore, since $\boldsymbol{B}$ and $\boldsymbol{A}(x)$ are upper triangular,

$$
\dot{A}_{i j}(x)=x^{-1} \sum_{k=i}^{j} B_{i k} A_{k j}(x), \quad x \in(0, \infty), \quad 1 \leqslant i \leqslant j \leqslant n .
$$


This system of differential equations can be solved as follows. If $i=j$, equation A.3 becomes

$$
\dot{A}_{i i}(x)=x^{-1} b_{i} A_{i i}(x), \quad x \in(0, \infty) .
$$

In combination with the initial condition $A_{i i}(1)=1$, this implies $A_{i i}(x)=x^{b_{i}}$.

Next assume $1 \leqslant i<j \leqslant n$. Rewrite A.3 as

$$
\dot{A}_{i j}(x)-x^{-1} b_{i} A_{i j}(x)=x^{-1} \sum_{k=i+1}^{j} B_{i k} A_{k j}(x), \quad x \in(0, \infty) .
$$

Looking for solutions of the form $A_{i j}(x)=C_{i j}(x) x^{b_{i}}$, we find that $C_{i j}(x)$ should satisfy

$$
\dot{C}_{i j}(x)=x^{-b_{i}-1} \sum_{k=i+1}^{j} B_{i k} A_{k j}(x), \quad x \in(0, \infty) .
$$

Since $C_{i j}(1)=0$, we obtain

$$
C_{i j}(x)=\sum_{k=i+1}^{j} B_{i k} \int_{1}^{x} A_{k j}(y) y^{-b_{i}-1} \mathrm{~d} y, \quad x \in(0, \infty)
$$

and consequently

$$
A_{i j}(x)=x^{b_{i}} \sum_{k=i+1}^{j} B_{i k} \int_{1}^{x} A_{k j}(y) y^{-b_{i}-1} \mathrm{~d} y, \quad x \in(0, \infty),
$$

which is A.1.

Next suppose that all diagonal elements of $\boldsymbol{B}$ are zero. Then all diagonal elements of $\boldsymbol{A}(x)$ are equal to unity, and by A.1,

$$
\begin{aligned}
A_{i, i+k}(x) & =\sum_{j=i+1}^{i+k} B_{i j} \int_{1}^{x} A_{j, i+k}(u) u^{-1} \mathrm{~d} u \\
& =\sum_{j=i+1}^{i+k-1} B_{i j} \int_{1}^{x} A_{j, i+k}(u) u^{-1} \mathrm{~d} u+B_{i, i+k} \log x .
\end{aligned}
$$

We proceed by induction on $k$. If $k=1$, then the above display tells us that $A_{i, i+1}(x)=$ $B_{i, i+1} \log x$, which is A.2. If $k \geqslant 2$, then use of the induction hypothesis and the previous display again leads, after some algebra, to the desired equality.

Remark A.2. (a) Let $\boldsymbol{B} \in \mathbb{R}^{n \times n}$ and write $\boldsymbol{H}(x)=\int_{1}^{x} u^{\boldsymbol{B}} u^{-1} \mathrm{~d} u$. Term-by-term integration of the series expansion $u^{B}=\sum_{k=0}^{\infty}(\log u)^{k} B^{k} / k$ ! yields the convenient formula

$$
\boldsymbol{B H}(x)=\boldsymbol{H}(x) \boldsymbol{B}=x^{\boldsymbol{B}}-\boldsymbol{I}, \quad x \in(0, \infty) .
$$

If $\boldsymbol{B}$ is invertible, then

$$
\boldsymbol{H}(x)=\boldsymbol{B}^{-1}\left(x^{\boldsymbol{B}}-\boldsymbol{I}\right)=\left(x^{\boldsymbol{B}}-\boldsymbol{I}\right) \boldsymbol{B}^{-1} .
$$

(b) Suppose that $\boldsymbol{D} \in \mathbb{R}^{(n+1) \times(n+1)}$ is given by

$$
\boldsymbol{D}=\left(\begin{array}{ll}
0 & \boldsymbol{c} \\
\mathbf{0} & \boldsymbol{B}
\end{array}\right)
$$


where $\boldsymbol{c} \in \mathbb{R}^{1 \times n}$ and where $\boldsymbol{B} \in \mathbb{R}^{n \times n}$ is upper triangular. Then $\boldsymbol{D}$ is upper triangular as well, and by Proposition A.1. for $x \in(0, \infty)$,

$$
x^{D}=\left(\begin{array}{cc}
1 & \boldsymbol{h}(x) \\
\mathbf{0} & x^{B}
\end{array}\right),
$$

where $\boldsymbol{h}(x) \in \mathbb{R}^{1 \times n}$ is given by

$$
\boldsymbol{h}(x)=\boldsymbol{c} \int_{1}^{x} y^{\boldsymbol{B}} y^{-1} \mathrm{~d} y .
$$

Acknowledgements. The research of the second author was partially supported by IAP research network grant no. P6/03 of the Belgian government (Belgian Science Policy).

\section{References}

N. H. Bingham and C. M. Goldie (1982a), Extensions of regular variation. I. Uniformity and quantifiers, Proc. London Math. Soc. (3) 44 (3), 473-496.

N. H. Bingham and C. M. Goldie (1982b), Extensions of regular variation. II. Representations and indices, Proc. London Math. Soc. (3) 44 (3), 497-534.

N. H. Bingham, C. M. Goldie, and J. L. Teugels (1987), Regular Variation, Cambridge University Press, Cambridge.

N. H. Bingham and A. J. Ostaszewski (2009), Infinite combinatorics and the foundations of regular variation, Journal of Mathematical Analysis and Applications 360 (2), 518-529.

E. W. Cheney and W. A. Light (2000), A Course in Approximation Theory, The Brooks/Cole Series in Advanced Mathematics, Brooks/Cole, Pacific Grove, CA.

S. Cheng and C. Jiang (2001), The Edgeworth expansion for distributions of extreme values, Science in China (Series A) 44, 427-437.

L. de Haan (1970), On regular variation and its application to the weak convergence of sample extremes, Mathematical Centre Tracts 32, Mathematisch Centrum, Amsterdam.

L. de Haan (1974), Equivalence classes of regularly varying functions, Stochastic Processes Appl. $2,243-259$.

L. de Haan and S. Resnick (1996), Second-order regular variation and rates of convergence in extreme-value theory, The Annals of Probability 24 (1), 97-124.

L. de Haan and U. Stadtmüller (1996), Generalized regular variation of second order, Journal of the Australian Mathematical Society (Series A) 61, 381-395.

H. Delange (1955), Sur un théorème de Karamata, Bull. Sci. Math. (2) 79, 9-12.

N. Dunford and J. T. Schwartz (1958), Linear Operators. I. General Theory, Pure and Applied Mathematics 7, Interscience, New York.

W. Feller (1971), An Introduction to Probability Theory and its Applications. Vol. II, 2nd ed., Wiley, New York.

I. Fraga Alves, L. de Haan, and T. Lin (2006), Third order extended regular variation, Publ. Inst. Math. (Beograd) (N.S.) 80 (94), 109-120.

J. L. Geluk and L. de Haan (1987), Regular variation, extensions and Tauberian theorems, Technical Report 40, CWI tract, Amsterdam.

C. M. Goldie and R. L. Smith (1987), Slow variation with remainder: theory and applications, Quart. J. Math. Oxford Ser. (2) 38 (149), 45-71. 
E. Haeusler and J. Segers (2007), Assessing confidence intervals for the tail index by Edgeworth expansions for the Hill estimator, Bernoulli 13 (1), 175-194.

E. Hille and R. S. Phillips (1974), Functional Analysis and Semi-groups, Amer. Math. Soc. Colloq. Publ. 31, Amer. Math. Soc., Providence, RI.

J. Karamata (1930), Sur un mode de croissance régulière des fonctions, Mathematica (Cluj) 4, $38-53$.

J. Karamata (1933), Sur un mode de croissance régulière. Théorèmes fondamentaux, Bull. Soc. Math. France 61, 55-62.

M. M. Meerschaert and H.-P. Scheffler (2001), Limit Distributions for Sums of Independent Random Vectors. Heavy Tails in Theory and Practice, Wiley, New York.

E. Omey and J. Segers (2009), Generalised regular variation of arbitrary order, Technical Report DP0901, Université catholique de Louvain, Louvain-la-Neuve; arXiv:0901.1468v1.

E. Omey and E. Willekens (1988), П-variation with remainder, J. London Math. Soc. (2) 37 (1), $105-118$

S. I. Resnick (1987), Extreme Values, Regular Variation, and Point Processes, Springer-Verlag, New York.

E. Seneta (1976), Regularly Varying Functions, Lecture Notes in Mathematics 508, SpringerVerlag, Berlin.

H. Steinhaus (1920), Sur les distances des points de mesure positive, Fund. Math. 1, 93-104.

X. Q. Wang and S. H. Cheng (2005), General regular variation of $n$-th order and the 2nd order Edgeworth expansion of the extreme value distribution. I. Acta Math. Sin. (Engl. Ser.) 21 (5), $1121-1130$

X. Q. Wang and S. H. Cheng (2006), General regular variation of the $n$-th order and 2nd order Edgeworth expansions of the extreme value distribution. II, Acta Math. Sin. (Engl. Ser.) 22 (1), 27-40 
